\title{
Martingais e \\ Teoria da Confiabilidade
}

\section{Hélio Arizono}

\author{
Dissertação Apresentada \\ $\mathrm{AO}$ \\ Instituto de Matemática e Estatística \\ DA \\ Universidade de São Paulo \\ para Obtenção do Grau de Mestre \\ EM \\ EsTATÍSTICA \\ Área de Concentração: Probabilidades \\ Orientador: Prof. Dr. Vanderlei da Costa Bueno \\ - SÃO PAULO, $1992-$
}




\section{ERRATA}

$\operatorname{Pg} 15$, linha 6: Trocar $Z_{V}^{f}(\emptyset) \Delta_{V}(\emptyset)$. por $Z_{V}^{f}(\emptyset) \Delta A_{V}(\emptyset)$.

$\operatorname{Pg} 21$, linha 18: $\mathbb{E}\left[S \mid \Im_{t}\right]$ por $\mathbb{E}\left[\tau \mid \Im_{t}\right]$

Pg 21, linha 19: $T_{i}, i=1, \ldots, n$ por $S_{i}, i=1, \ldots, n$

Pg 23, linha 6: $I_{v}(\tau, \tau) d v$ por $I_{v}^{B}(\tau, \tau) d v$

$\operatorname{Pg} 26$, linha 13: $\exp \left[i u_{j} A_{T_{j}}(j)\right]$ por $\exp \left[i u_{j} A_{S_{j}}(j)\right]$

Pg 26, linha 15: $\mathbb{E}\left\{\exp \left[\sum_{j=1}^{n} i u_{j} A_{T_{j}}(j)\right]\right\}$ por $\mathbb{E}\left\{\exp \left[\sum_{j=1}^{n} i u_{j} A_{S_{j}}(j)\right]\right\}$

Pg 28, linha 11: $\ln \bar{F}_{i}(t) \bar{F}_{i}(t)+\bar{F}_{i}(t)$ por $\ln \bar{F}_{i}(t) \bar{F}_{i}(t)+F_{i}(t)$

Pg 28, linha 14: $\left(\ln \bar{F}_{i}(t) \bar{F}_{i}(t)+\bar{F}_{i}(t)\right) \operatorname{por}\left(\ln \bar{F}_{i}(t) \bar{F}_{i}(t)+F_{i}(t)\right)+$

Pg 34, linha 13: $\frac{P\{T=t\}}{F[t, \infty)}$ por $\frac{P\{\tau=t\}}{F[t, \infty)}$

$\mathrm{Pg}$ 36, linha 22: 'super-martingal' por 'sub-martingal'

Pg 43, linha 2: $\tau=\max _{1 \leq k \leq k_{0}} \min _{i \in P_{k}} T_{i}$ por $\tau=\max _{1 \leq k \leq k_{0}} \min _{i \in P_{k}} S_{i}$

$\operatorname{Pg} 43$, linha $7: \mathbb{P}\left\{\theta_{t} \mathrm{~S} \in U\right\}=\mathbb{P}\left\{\theta_{t} \tau>d\right\}$ por $\mathbb{P}\left\{\theta_{t} \mathrm{~S} \in U \mid \Im_{t}\right\}=\mathbb{P}\left\{\theta_{t} \tau>d \mid \Im_{t}\right\}$

Pg 43, linha 8: $\mathbb{P}\left\{\theta_{t^{*}} \tau>d\right\}=\mathbb{P}\left\{\theta_{t^{*}} \mathrm{~S} \in U\right\}$ por $\mathbb{P}\left\{\theta_{t^{*}} \tau>d \mid \Im_{t}\right\}=\mathbb{P}\left\{\theta_{t^{*}} \mathrm{~S} \in U \mid \Im_{t}\right\}$

$\operatorname{Pg}$ 47, linha 9: $\mathbf{T}_{\mathbf{2}}$ por $\mathbf{S}_{\mathbf{2}}$

$\operatorname{Pg}$ 54, linha 10: $\Leftrightarrow\left\{T \wedge T_{k}<s\right\}$. por $\Leftrightarrow\left\{T \wedge T_{k+1}<s\right\}$.

Pg 56, linha 4: $\int_{0}^{\left(T_{k+1} \wedge T_{k}\right)+R_{k}-T_{k}}$ por $\int_{0}^{T_{k+1} \wedge\left(T_{k}+R_{k}\right)-T_{k}}$

Pg 62, linha 6: $\mathbb{P}\left((\tau-t)^{+}>\mid \Im_{t}\right)$ por $\mathbb{P}\left((\tau-t)^{+}>u \mid \Im_{t}\right)$ æ 


\section{Agradecimentos}

ao Prof. Dr. Vanderlei da Costa Bueno. pela amizade e pela atenciosa orientação,

à Vera. pela dedicaçào na revisão desta dissertação .

a Rinaldo Artes, pelas dicas e pelo arquivo do $\mathrm{T}_{\mathrm{EX}}$,

aos meus amigos Andrea. Clándia e Ernesto. pela ajuda no traballo.

aos meus pais e minha immà. pela paciência e complucensäo. 


\section{RESUMO: MARTINGAIS E TEORIA DA CONFIABILIDADE}

Frequentemente os problemas que aparecem na Teoria da Confiabilidade são resolvidos por cálculos da confiabilidade de natureza estática: a variável de tempo $t$ é usualmente fixada e a evolução da confiabilidade do sistema no tempo é ignorada. Uma abordagem simples para considerar a dinâmica do sistema no tempo e a interdependência entre os componentes se faz com o uso da Teoria dos Martingais para Processos Pontuais.

Nessa dissertação determinamos o processo pontual multivariado das falhas dos componentes de um sistema, caracterizamos a propriedade de associação de variáveis aleatórias através dos Processos Covariância, analisamos medidas de importância da confiabilidade do componente, generalizamos a noção de função de risco e estudamos classes de distribuições de vida em termos da ordenação estocástica condicional a $\sigma$-álgebra do passado observado.

\section{ABSTRACT: MARTINGALES AND RELIABILITY THEORY}

Usually problems in reliability theory are solved by reliability calculations of static nature: the time variable $t$ is usually a fixed value and the evolution of the system reliability in time is ignored. A simple approach. considering the dynamic of the system in time and the interdependence between components. uses Martingale Theory for Point Process.

In this dissertation we consider the multivariate point process of component's system failure, characterize the association property of random variables through Covariance Processes, analize the reliability importance measure of components, generalize the risk function notion and study the life distributions classes in terms of conditional stochastic order given the $\sigma$-field corresponding to observed past. 


\section{Conteúdo}

Introdução

Capítulo 1. ASSOCIAÇÃO E IMPORTÂNCIA DOS COMPONENTES.

1.1 O Processo Pontual Multivariado das Falhas dos componentes

1.2 Associação dos Componentes

1.3 Importância de Componentes 20

Capítulo 2. O PROCESSO DE RISCO DOS COMPONENTES E DO SISTEMA

2.1 A Função de Risco através do Compensador

2.2 Classes de Distribuições $\operatorname{MIFR} \mid\left(\Im_{t}\right)$

2.3 Classes de Distribuições MNBU|( $\left.\Im_{t}\right)$

2.4 O Processo de Risco

Apêndices

A.1 Ordenação Estocástica.

A.2 Compensadores Calculáveis

Bibliografia 
INTRODUÇÃO

$$
-1 \text { - }
$$


Frequentemente os problemas que aparecem na Teoria da Confiabilidade são resolvidos por cálculos da confiabilidade de natureza estática: a variável de tempo $t$ é usualmente fixada e a evolução da confiabilidade do sistema no tempo é ignorada. Além disso, uma avaliação realista do grau de dependência entre os componentes, em situações práticas, é geralmente extremamente complicada e de formulação matemática muito difícil.

Apesar das razões acima, uma abordagem simples para considerar a dinâmica do sistema no tempo e a interdependência entre os componentes se faz com o uso da Teoria dos Martingais para Processos Pontuais:

Considere um sistema $\phi$ com tempo de vida $\tau$, de $n$ componentes $C_{1}, C_{2}, \ldots, C_{n}$, com tempos de vida $S_{1}, S_{2}, \ldots, S_{n}$, respectivamente. Ao invés de analisarmos a distribuição conjunta de $\mathrm{S}=\left(S_{1}, \ldots, S_{n}\right)$, analisaremos os processos de contagem associados com as falhas dos componentes,

$$
N_{t}(i)=1_{\left\{S_{i} \leq t\right\}}, \quad i=1, \ldots, n
$$

e o processo de contagem da falha do sistema $N_{t}(\phi)=1_{\{\tau \leq t\}}$.

Suponha que o comportamento do sistema é monitorado ao nível dos componentes, isto é, a cada tempo $t$ e para todo componente $i$, o pesquisador conhece se o evento $\left\{S_{i} \leq t\right\}$ ocorreu ou não e se $\left\{S_{i} \leq t\right\}$ ocorreu, ele sabe o exato valor de $S_{i}$. A formulação matemática deste conhecimento é a história gerada pelos indicadores dos componentes

$$
\Im_{t}=\sigma\left\{N_{s}(i), s \leq t, i=1, \ldots, n\right\} .
$$

A dinâmica da confiabilidade do sistema pode ser considerada pela evolução no tempo dos valores esperados condicionais

$$
\mathbb{E}\left[f(\mathrm{~S}) \mid \Im_{t}\right]
$$

para funções $f: \mathbb{R}_{+}^{n} \rightarrow \mathbb{R}^{1}$ convenientemente escolhidas.

Uma escolha natural para $f$ seria a função de estrutura $\phi$ e, neste caso, o martingal uniformemente integrável $\left(\mathbb{E}\left[\phi(\mathrm{S}) \mid \Im_{t}\right]\right)_{t \geq 0}$ descreve como o tempo de vida estimado do 
sistema evolui com o tempo, condicionalmente ao nível de informação obtida através da observação dos componentes até o instante $t$.

O conceito de Associação entre os componentes pode ser analisado através do processo de covariância $\left(<\mathbb{E}\left[f(\mathbf{S}) \mid \Im_{t}\right], \mathbb{E}\left[g(\mathbf{S}) \mid \Im_{t}\right]>\right)_{t \geq 0}$ onde $f$ e $g$ são duas funções crescentes, limitadas e Borél mensuráveis definidas em $\mathbb{R}_{+}^{n}$, com valores em $\mathbb{R}$.

A Importância da Confiabilidade de Natvig do componente $i$ pode ser obtida através da redução esperada do martingal $\left(\mathbb{E}\left[\phi(\mathbf{S}) \mid \Im_{t}\right]\right)_{t \geq 0}$, no tempo $S_{i}$, em que o componente $i$ falha, isto é,

$$
I^{N}\left(\tau, S_{i}\right)=\mathbb{E}\left\{\mathbb{E}\left[\phi(\mathbf{S}) \mid \Im_{S_{i}}\right]-\mathbb{E}\left[\phi(\mathbf{S}) \mid \Im_{S_{i}-}\right]\right\}
$$

Ao considerarmos um objeto com tempo de vida $\tau$, com função de distribuição $F$, a função de risco $R(t)$ é definida por

$$
R(t)=\int_{0}^{t} \frac{d F(s)}{F[s, \infty)}
$$

Pode-se estudar algumas classes de distribuições úteis na Teoria da Confiabilidade através da função de risco. Por exemplo, se $F$ é absolutamente contínua:

- F tem taxa de falha crescente (IFR) se e somente se $R(t)$ é convexa;

- F tem a propriedade NBU se e somente se $R(t+s) \geq R(t)+R(s)$, para todo $t$ e $s \geq 0$.

No conjunto $\{t<S\}$ a função de risco coincide com o $\left(\Im_{t}\right)$-compensador do processo de contagem $1_{\{r \leq t\}}$, onde $\Im_{t}=\sigma\left\{1_{\{\tau \leq s\}}, s \leq t\right\}$. Ao tratarmos a dinâmica de vários componentes simultaneamente podemos generalizar a função de risco através dos $\left(\Im_{t}\right)$-compensadores chegando assim ao Processo de Risco, que dependerá fundamentalmente da família de $\sigma$-álgebras $\left(\Im_{t}\right)_{t \geq 0}$. Consequentemente, para analisarmos a relação entre o Processo de Risco e as Classes de Distribuições devemos considerá-las condicionalmente à família de $\sigma$-álgebras $\left(\Im_{t}\right)_{t \geq 0}$.

Nessa dissertação, no primeiro capítulo, determinaremos o processo pontual multivariado das falhas dos componentes de um sistema, caracterizaremos a propriedade de 
associação de variáveis aleatórias através do processo de covariância e analisaremos medidas de importância da confiabilidade do componente. No segundo capítulo, generalizaremos a noção da função de risco e estudaremos classes de distribuições de vida em termos da ordenação estocástica condicional a $\sigma$-álgebra do passado observado. 
CAPÍTULO 1

CAPÍTULO 1: ASSOCIAÇÃO E IMPORTÂNCIA DOS COMPONENTES 


\subsection{O Processo Pontual Multivariado das Falhas dos Componentes.}

Consideremos uma coleção de $n$ componentes $C_{1}, C_{2}, \ldots, C_{n}$ que compõem um sistema complexo $\phi$. Cada componente $C_{i}$ tem um tempo de vida aleatório e positivo $S_{i}, 1 \leq i \leq n$, definidas em um espaço de probabilidade $(\Omega, \Im, P)$. Interpretaremos o instante inicial 0 (zero) como o tempo em que $\phi$ começa a funcionar.

A todo instante $t$, o pesquisador sabe se o evento $\left\{S_{i} \leq t\right\}, 1 \leq i \leq n$, ocorreu ou não e caso tenha ocorrido, ele conhece exatamente o valor de $S_{i}$. A formulação matemática desse conhecimento até o instante $t$ é obtida através da família

$$
\Im_{t}=\sigma\left\{1_{\left\{S_{i} \leq s\right\}}, s \leq t ; 1 \leq i \leq n\right\}
$$

de $\sigma$-álgebras crescentes, contínuas à direita e completadas com os conjuntos de medida nula de $\left(\Im_{t}\right)_{t \geq 0}$.

As falhas de $C_{1}, \ldots, C_{n}$ aparecem no decorrer do tempo como um processo estocástico e este fato é interpretado convenientemente através de um processo pontual multivariado. Para determinada realização $\omega$ fixada, seja $q(\omega)$ o número de valores distintos no conjunto $\left\{S_{i}(\omega), 1 \leq i \leq n\right\}$. As estatísticas de ordem crescentes desse conjunto denotaremos por:

$$
T_{1}(\omega)<T_{2}(\omega)<\ldots<T_{q(\omega)}(\omega)
$$

e definiremos também o conjunto dos componentes que falham no $k$-ésimo tempo de falha $T_{k}$, por

$$
X_{k}(\omega)=\left\{i: 1 \leq i \leq n, S_{i}(\omega)=T_{k}(\omega)\right\}
$$

Se não existirem falhas múltiplas, o valor de $X_{k}$ é um dos conjuntos $\{i\}, 1 \leq i \leq n$, mas em geral, $X_{k}$ é uma variável aleatória com valores em $\Lambda=\mathcal{P}\{1,2, \ldots, n\}$, o conjunto das partes de $\{1,2, \ldots, n\}$.

Chamaremos $T_{k}$ o $k$-ésimo tempo de falha e $X_{k}$ o $k$-ésimo conjunto de falha. 
Assim, a sequência aleatória $\left(T_{k}, X_{k}\right)_{1 \leq k \leq q}$ descreve como os componentes $C_{1}, \ldots, C_{n}$ falharam e considerando $T_{q+1}=T_{q+2}=\ldots=\infty$ e $X_{q+1}=X_{q+2}=\ldots=\emptyset$ podemos definir:

Definição 1.1.1: Definimos o Processo de Falhas dos Componentes como o processo pontual multivariado $\left(T_{k}, X_{k}\right)_{k \geq 1}$.

Uma maneira equivalente de descrever esse processo é através dos processos de contagem: para cada conjunto de falha $I \in \Lambda$ fixado, sejam $\tau_{I}$ e $N_{t}(\omega ; I)$ definidos por

$$
\begin{gathered}
\tau_{I}=\inf \left\{T_{k}: X_{k}=I\right\} \quad(\inf \emptyset=\infty) \quad e \\
N_{t}(\omega ; I)= \begin{cases}0, & \text { se } t<\tau_{I}(\omega), \\
1, & \text { se } t \geq \tau_{I}(\omega) .\end{cases}
\end{gathered}
$$

Para cada $\omega,\left(S_{i}(\omega)\right)_{1 \leq i \leq n}$ determina a realização do processo $\left(N_{t}(I) ; I \in \Lambda\right)_{t \geq 0} \mathrm{e}$ vice-versa. Similarmente

$$
\Im_{t}=\sigma\left\{1_{\left\{S_{i} \leq s\right\}} ; s \leq t, 1 \leq i \leq n\right\} \text { é equivalente a } \Im_{t}=\sigma\left\{N_{s}(I) ; s \leq t, I \in \Lambda\right\} .
$$

Com o conhecimento acumulado $\left(\Im_{t}\right)_{t \geq 0}$ da evolução dos componentes no tempo, podemos observar o comportamento no tempo das esperanças condicionais $E\left[f(\mathrm{~S}) \mid \Im_{t}\right]$, para funções mensuráveis $f$ convenientemente escolhidas. Para $f$ fixada, assumiremos que $E\left[f(\mathrm{~S}) \mid \Im_{t}\right]$ é um martingal contínuo à direita com limites à esquerda.

O tempo de vida $\tau$, do sistema $\phi$, é completamente determinado pelos tempos de vida dos componentes através da relação funcional

$$
\begin{aligned}
\tau=\phi(\mathrm{S}) & =\min _{1 \leq j \leq k} \max _{i \in K_{j}} S_{i} \\
& =\max _{1 \leq j \leq p} \min _{i \in P_{j}} S_{i},
\end{aligned}
$$

onde $K_{1}, \ldots, K_{k}$ são os conjuntos de cortes e $P_{1}, \ldots, P_{p}$ são os conjuntos de caminhos da estrutura $\phi$ (Barlow e Proschan (1981)).

Definição 1.1.2: Dado um sistema $\phi$ de componentes $C_{1}, C_{2}, \ldots, C_{n}$, definimos sua função de estrutura como 


\section{$E\left[\phi(\mathbf{S}) \mid \Im_{t}\right](\omega)$}

onde $\mathbf{S}=\left(S_{1}, \ldots, S_{n}\right)$ e $S_{i}, 1 \leq i \leq n$, são os tempos de vida dos componentes.

Neste caso, o martingal $\left(E\left[\phi(\mathrm{S}) \mid \Im_{t}\right]\right)_{t \geq 0}$ descreve como o tempo de vida estimado do sistema evolui com o tempo, dado as observações dos componentes até o instante $t$.

Algumas propriedades estruturais simples são facilmente reproduzidas, por exemplo:

a) Redundâncias aos níveis dos componentes é mais recomendável do que redundância ao nível do sistema, isto é:

$$
E\left[\phi(\mathbf{S} \vee \mathbf{R}) \mid \Im_{t}\right] \geq E\left[\phi(\mathbf{S}) \mid \Im_{t}\right] \vee E\left[\phi(\mathbf{R}) \mid \Im_{t}\right]
$$

onde $\mathrm{S}=\left(S_{1}, \ldots, S_{n}\right), \mathbf{R}=\left(R_{1}, \ldots, R_{n}\right)$,

$$
\begin{aligned}
& \mathrm{S} \vee \mathbf{R}=\left(S_{1} \vee R_{1}, \ldots, S_{n} \vee R_{n}\right) \mathrm{e} \\
& S_{i} \vee R_{i}=\max \left\{S_{i}, R_{i}\right\}, 1 \leq i \leq n .
\end{aligned}
$$

b) O desempenho de um sistema qualquer é sempre melhor do que o de um sistema em série e pior do que um sistema em paralelo, isto é:

$$
\mathbb{E}\left[\min _{1 \leq i \leq n} S_{i} \mid \Im_{t}\right] \leq E\left[\phi(\mathrm{S}) \mid \Im_{t}\right] \leq E\left[\max _{1 \leq i \leq n} S_{i} \mid \Im_{t}\right]
$$

Definição 1.1.3: A confiabilidade do sistema $\phi$, de componentes $C_{1}, \ldots, C_{n}$, com tempos de vida $S_{1}, \ldots, S_{n}$, respectivamente, é

$$
E[\phi(\mathrm{S})]=E\left\{E\left[\phi(\mathrm{S}) \mid \Im_{t}\right]\right\}
$$

Exemplo 1.1.3: Seja $\phi$ um sistema formado por dois componentes independentes em paralelo com distribuições exponenciais de parâmetro 1. Então $\phi(\mathrm{S})=S_{1} \vee S_{2}$ e se consideramos $\Im_{t}=\sigma\left\{1_{\left\{S_{i} \leq s\right\}}, s \leq t, i=1,2\right\}$ temos que 


$$
\mathbb{E}(\phi(\mathrm{S}))=\int_{0}^{\infty} \mathbb{P}(\tau>t) d t=1.5
$$

Para $s$ e $t \geq 0$

$$
\begin{aligned}
\mathbb{P}\left(\phi(\mathrm{S}) \leq t+s \mid S_{1}>s, S_{2}>s\right) & =\frac{\mathbb{P}\left(s<S_{1} \leq t+s, s<S_{2} \leq t+s\right)}{\mathbb{P}\left(S_{1}>s, S_{2}>s\right)} \\
& =\mathbb{P}\left(S_{1} \leq t+s \mid S_{1}>s\right) \mathbb{P}\left(S_{2} \leq t+s \mid S_{1}>s\right) \\
& =\mathbb{P}\left(S_{1} \leq t\right) \mathbb{P}\left(S_{2} \leq t\right) \\
& =\mathbb{P}(\phi(\mathrm{S}) \leq t) .
\end{aligned}
$$

A distribuição condicional de $\phi(\mathbf{S})-s$ dado que $T_{1}=s$ é $\exp (1)$.

Assim, por (1.1.1), (1.1.2) e (1.1.3), obtemos que

$$
\mathbb{E}\left(\phi(\mathrm{S}) \mid \Im_{t}\right)= \begin{cases}t+1.5, & \text { se } 0 \leq t<T_{1} \\ t+1, & \text { se } T_{1} \leq t<T_{2} \\ T_{2}, & \text { se } t \geq T_{2}\end{cases}
$$

$\mathrm{Na}$ maioria das vezes, os sistemas na engenharia são por demais complexos e na presença de dependência entre os componentes o cálculo da confiabilidade do sistema é praticamente impossível. Torna-se necessário definir limites superiores e inferiores para a confiabilidade dos sistemas complexos. No caso clássico, estes limites são naturalmente obtidos na presença de associação entre os componentes, que é uma forma de dependência positiva. O vetor $\mathbf{R}=\left(R_{1}, \ldots, R_{n}\right)$ é associado se e somente se $\operatorname{Cov}(f(\mathbf{R}), g(\mathbf{R})) \geq 0$, para todas as funções $g$ e $f$ crescentes e limitadas, definidas em $\mathbb{R}^{n}$ e com valores em $\mathbb{R}$ (Barlow e Proschan (1981)). 


\subsection{Associação dos Componentes.}

Considere para quaisquer funções limitadas e mensuráveis $f$ e $g$, versões contínuas à direita com limites à esquerda dos martingais $M_{t}^{f}=E\left[f(\mathbf{S}) \mid \Im_{t}\right], M_{t}^{g}=E\left[g(\mathbf{S}) \mid \Im_{t}\right]$ e o processo covariância $\left.\left(<M^{f}, M^{g}\right\rangle_{t}\right)_{t \geq 0}$ definido por:

Definição 1.2.1: O processo covariância $\left.\left(<M^{f}, M^{g}\right\rangle\right)_{t \geq 0}$ é o único processo previsível, de variação limitada, com $<M^{f}, M^{g}>_{0}=0$ e tal que

$$
M_{t}^{f} M_{t}^{g}-<M^{f}, M^{g}>_{t}
$$

é um martingal.

Proposição 1.2.2: Nas condições acima, vale

$$
E<M^{f}, M^{g}>_{\infty}=\operatorname{Cov}(f(\mathrm{~S}), g(\mathrm{~S})) .
$$

Prova: Interpretaremos $\Im_{0}$ como sendo a $\sigma$-álgebra trivial $\sigma\{\emptyset, \Omega\}$. Assim, $E\left[g(\mathbf{S}) \mid \Im_{0}\right]=E[g(\mathrm{~S})]$ e $E\left[M_{0}^{f} M_{0}^{g}\right]=E[f(\mathrm{~S})] E[g(\mathrm{~S})]$. Como $M_{t}^{f} M_{t}^{g}-<M^{f}, M^{g}>_{t}$ é um $\left(\Im_{t}\right)_{t \geq 0}-$ martingal e tem esperança constante, concluímos que:

$$
E\left[M_{t}^{f} M_{t}^{g}\right]-E\left[<M^{f}, M^{g}>_{t}\right]=E\left[M_{0}^{f} M_{0}^{g}\right]=E[f(\mathbf{S})] E[g(\mathbf{S})],
$$

de maneira que

$$
\begin{aligned}
E\left[<M^{f}, M^{g}>_{\infty}\right] & =E\left\{E\left[f(\mathbf{S}) \mid \Im_{\infty}\right] E\left[g(\mathbf{S}) \mid \Im_{\infty}\right]\right\}-E[f(\mathbf{S})] E[g(\mathbf{S})] \\
& =E[f(\mathbf{S}) g(\mathbf{S})]-E[f(\mathbf{S})] E[g(\mathbf{S})] \\
& =\operatorname{Cov}(f(\mathbf{S}), g(\mathbf{S})) .
\end{aligned}
$$

Em decorrência da Proposição 1.2.2 podemos usar um conceito alternativo (Arjas e Norros (1984)) para um vetor aleatório associado: 
Definição 1.2.3: O vetor aleatório $S$ é associado se e somente se para todas funções $f$ e $g$ limitadas, mensuráveis e crescentes definidas em $\mathbb{R}^{n}$ com valores em $\mathbb{R}$, temos:

$$
E\left[<M^{f}, M^{g}>_{\infty}\right] \geq 0
$$

No que segue, caracterizaremos o processo $\left\langle M^{f}, M^{g}\right\rangle_{t}$ e procuraremos condições para que $E\left[<M^{f}, M^{g}>\infty\right] \geq 0$. Para tanto, observemos que, para cada $I \in \Lambda$, o processo pontual $N_{t}(I)=1_{\left\{\tau_{I} \leq t\right\}}$ admite a decomposição de Doob-Meyer

$$
N_{t}(I)=A_{t}(I)+M_{t}(I)
$$

onde $A_{t}(I)$ é o único processo natural crescente e contínuo à direita, com $A_{0}(I)=0$ (o $\left(\Im_{t}\right)_{t \geq 0}$-compensador de $\left.N_{t}(I)\right)$ tal que $M_{t}(I)=N_{t}(I)-A_{t}(I)$ é um $\left(\Im_{t}\right)$-martingal.

Proposição 1.2.4: O processo covariância $\left.\left(<M^{f}, M^{g}\right\rangle_{t}\right)_{t \geq 0}$ tem a representação

$$
<M^{f}, M^{g}>_{t}=\sum_{I \in \Lambda} \int_{0}^{t} C_{s}^{f}(I) C_{s}^{g}(I) d A_{s}^{c}(I)+\sum_{s \leq t} \operatorname{Cov}_{\Delta A}\left(C_{s}^{f}(\cdot), C_{s}^{g}(\cdot)\right)
$$

onde $A^{c}(I)$ é a parte contínua de $A_{t}(I)$;

$$
\begin{aligned}
& C^{f}(I)=\left(C_{t}^{f}(I)\right)_{t \geq 0} \text { e } C^{g}(I)=\left(C_{t}^{g}(I)\right)_{t \geq 0} \text { são previsíveis e } \\
& \begin{aligned}
\operatorname{Cov}_{\Delta A}\left(C_{s}^{f}(\cdot), C_{s}^{g}(\cdot)\right)= & \sum_{I \in \Lambda} C_{s}^{f}(I) C_{s}^{g}(I) \Delta A_{s}(I)- \\
& \quad-\left(\sum_{I \in \Lambda} C_{s}^{f}(I) \Delta A_{s}(I)\right)\left(\sum_{I \in \Lambda} C_{s}^{g}(I) \Delta A_{s}(I)\right) .
\end{aligned}
\end{aligned}
$$

Prova: Utilizando a decomposição integral para martingais, podemos representar $M_{t}^{f}$ como

$$
\begin{aligned}
M_{t}^{f}=E[f(\mathrm{~S})] & +\sum_{I \in \Lambda} \int_{0}^{t} C_{s}^{f}(I) d M_{s}(I) \\
& -11-
\end{aligned}
$$


onde o processo $\left(C_{t}^{f}(I)\right)_{t \geq 0}$ é previsível e $\left(M_{t}(I)\right)_{t \geq 0}$ é o martingal resultante da decomposição de Doob-Meyer.

Segue que

$$
<M^{f}, M^{g}>_{t}=\sum_{I, J \in \Lambda} \int_{0}^{t} C_{s}^{t}(I) C_{s}^{g}(J) d<M(I), M(J)>_{s} .
$$

Contudo, pela regra de diferenciação de Stieltjes, temos

$$
\begin{aligned}
M_{t}(I) M_{t}(J)= & \int_{0}^{t} M_{s^{-}}(I) d M_{s}(J)+\int_{0}^{t} M_{s^{-}}(J) d M_{s}(I)+ \\
& +\sum_{s \leq t}\left(\Delta N_{s}(I)-\Delta A_{s}(I)\right)\left(\Delta N_{s}(J)-\Delta A_{s}(J)\right) \\
= & \int_{0}^{t} M_{s^{-}}(I) d M_{s}(J)+\int_{0}^{t} M_{s^{-}}(J) d M_{s}(I)+ \\
& +\sum_{s \leq t}\left(\Delta N_{s}(I)-\Delta A_{s}(I)\right) \Delta N_{s}(J)-\sum_{s \leq t}\left(\Delta N_{s}(I)-\Delta A_{s}(I)\right) \Delta A_{s}(J) \\
= & \int_{0}^{t} M_{s^{-}}(I) d M_{s}(J)+\int_{0}^{t} M_{s^{-}}(J) d M_{s}(I)+ \\
& +\int_{0}^{t}\left(\delta_{I J}-\Delta A_{s}(I)\right) d N_{s}(J)-\int_{0}^{t} \Delta A_{s}(J) d M_{s}(I) \\
= & {\left[\int_{0}^{t} M_{s^{-}}(I) d M_{s}(J)+\int_{0}^{t} M_{s^{-}}(J) d M_{s}(I)+\int_{0}^{t}\left(\delta_{I J}-\Delta A_{s}(I)\right) d M_{s}(J)-\right.} \\
& \left.-\int_{0}^{t} \Delta A_{s}(J) d M_{s}(I)\right]+\int_{0}^{t}\left(\delta_{I J}-\Delta A_{s}(I)\right) d A_{s}(J) .
\end{aligned}
$$

Como $M_{s^{-}}(I), M_{s^{-}}(J), \Delta A_{s}(J), \delta_{I J}-\Delta A_{s}(I)$ são previsíveis, concluímos que as integrais entre colchetes são martingais. Sendo o processo $\int_{0}^{t}\left(\delta_{I J}-\Delta A_{s}(I)\right) d A_{s}(I)$ previsível, de variação limitada e nulo no ponto 0 , concluímos, pela unicidade do processo covariância, que

$$
<M(I), M(J)>_{t}=\int_{0}^{t}\left(\delta_{I J}-\Delta A_{s}(I)\right) d A_{s}(J)
$$

e portanto 


$$
d<M(I)<M(J)>_{t}=\left(\delta_{I J}-\Delta A_{t}(I)\right) d A_{t}(J)
$$

Substituindo essa expressão em (1.2.4), obtemos

$$
\begin{aligned}
<M^{f}, M^{g}>_{t}= & \sum_{I, J \in \Lambda} \int_{0}^{t} C_{s}^{f}(I) C_{s}^{g}(J)\left(\delta_{I, J}-\Delta A_{s}(I)\right) d A_{s}(J) \\
= & \sum_{I, J \in \Lambda} \int_{0}^{t} C_{s}^{f}(I) C_{s}^{g}(I) \delta_{I J} d A_{s}(J)-\sum_{I, J \in \Lambda} \int_{0}^{t} C_{s}^{f}(I) C_{s}^{g}(J) \Delta A_{s}(I) d A_{s}(J) \\
= & \sum_{I \in \Lambda} \int_{0}^{t} C_{s}^{f}(I) C_{s}^{g}(I) d A_{s}^{c}(I)+\sum_{s \leq t} \sum_{I \in \Lambda} C_{s}^{f}(I) C_{s}^{g}(I) \Delta A_{s}(I)- \\
& -\sum_{s \leq t}\left(\sum_{I \in \Lambda} C_{s}^{f}(I) \Delta A_{s}(I)\right)\left(\sum_{I \in \Lambda} C_{s}^{g}(I) \Delta A_{s}(I)\right) \\
= & \sum_{I \in \Lambda} \int_{0}^{t} C_{s}^{f}(I) C_{s}^{g}(I) d A_{s}^{c}(I)+\sum_{s \leq t} \operatorname{Cov}_{\Delta A}\left(C_{s}^{f}(\cdot), C_{s}^{g}(\cdot)\right)
\end{aligned}
$$

Convenientemente, para $I=\emptyset$ definiremos $\Delta A_{t}(\emptyset)=1-\sum_{I \in \Lambda} \Delta A_{t}(I), t \geq 0$ de maneira que para cada $t$ fixado, $\left(\triangle A_{t}(I), I \in \Lambda^{\circ}=\Lambda \cup \emptyset\right)$ é uma medida de probabilidade em $\Lambda^{o}$. As frequências $\Delta A_{t}(I), I \in \Lambda^{o}$ podem ser interpretadas como as probabilidades de se obter a falha do conjunto $I$ no tempo $t$, condicionado a $\Im_{t^{-}}$enquanto $\Delta A_{t}(\emptyset)$ corresponde à probabilidade de nenhuma falha em $t$.

Definindo $C_{s}^{f}(\emptyset)=C_{s}^{g}(\emptyset)=0$ podemos interpretar $\operatorname{Cov}_{\Delta A}\left(C_{s}^{f}(\cdot), C_{s}^{g}(\cdot)\right)$ como a $\operatorname{Cov}\left(C_{s}^{f}(D), C_{s}^{g}(D)\right)$ onde $D$ é uma variável aleatória com valores em $\Lambda^{o}$ e distribuição de probabilidades $\left(\triangle A_{s}(I), I \in \Lambda^{\circ}\right)$.

Para prosseguirmos a caracterização do processo covariância necessitaremos de uma forma explícita para os processos $C^{f}(I)$ e $C^{g}(I)$. Tal resultado é formulado na Proposição 1.2.6.

Como $\Im_{T_{k}^{-}}=\sigma\left\{T_{1}, X_{1}, T_{2}, X_{2}, \ldots, T_{k-1}, X_{k-1}, T_{k}\right\}, T_{k}$ é $\Im_{T_{k}^{-}}-$mensurável e desde de que $\tau_{I}=T_{k}$, para algum $k$ no conjunto $\left\{\tau_{I}<\infty\right\}$, concluímos que existem funções $\varphi_{I}^{k}$ tais que 


$$
M_{\tau_{I}}^{f} 1_{\left\{\tau_{I} \leq \infty\right\}}=\sum_{k \geq 1} 1_{\left\{\tau_{I}=T_{k}\right\}} \varphi_{I}^{k}\left(T_{1}, X_{1}, \ldots, T_{k-1}, X_{k-1}, T_{k}\right)
$$

e podemos então considerar o processo $Z^{f}(I)=\left(Z_{t}^{f}(I)\right)_{t \geq 0}, I \in \Lambda$ tal que

$$
Z_{t}^{f}(I)=\sum_{k \geq 1} 1_{\left\{t \in\left(T_{k-1}, T_{k}\right]\right\}} \varphi_{I}^{k}\left(T_{1}, X_{1}, \ldots, T_{k-1}, X_{k-1}, t\right)
$$

de maneira que, para cada $I \in \Lambda, Z^{f}(I)$ é previsível e

$$
Z_{\tau_{I}}^{f}(I) 1_{\left(\tau_{I}<\infty\right)}=M_{\tau_{I}}^{f} 1_{\left(\tau_{I}<\infty\right)}
$$

Definindo de maneira conveniente

$$
Z_{t}^{f}(\emptyset)=\frac{M_{t^{-}}^{f}-\sum_{I \in \Lambda} Z_{t}^{f}(I) \Delta A_{t}(I)}{\Delta A_{t}(\emptyset)} 1_{\left\{\Delta A_{t}(\emptyset)>0\right\}}+\sup _{x \in \mathbb{R}^{k}}|f(\mathrm{x})| 1_{\left\{\Delta A_{t}(\emptyset)=0\right\}}
$$

podemos demonstrar o seguinte lema

Lema 1.2.5: Para todo $t \geq 0$,

$$
M_{t^{-}}^{f}=\sum_{I \in \Lambda^{\circ}} Z_{t}^{f}(I) \Delta A_{t}(I)
$$

Prova: Se $\Delta A_{t}(\emptyset)>0$, o resultado é imediato. Em geral, para um tempo de parada previsível $V$, existe uma sequência $\left(V_{n}\right)_{n \geq 1}$ de tempos de paradas crescentes, tal que $V_{n}<V, n \geq 1$ com $\lim _{n \rightarrow \infty} V_{n}=V$, de maneira que

$$
\begin{aligned}
M_{V-}^{f} & =\lim _{n} M_{V_{n}}^{f}=\lim _{n} \mathbb{E}\left[f(\mathbf{S}) \mid \Im_{V_{n}}\right] \\
& =\mathbb{E}\left[f(\mathrm{~S}) \mid \bigvee_{n} \Im_{V_{n}}\right] \\
& =\mathbb{E}\left[f(\mathrm{~S}) \mid \Im_{V^{-}}\right] .
\end{aligned}
$$

Assim, 


$$
\begin{aligned}
M_{V^{-}}^{f} & =\mathbb{E}\left[M_{V}^{f} \mid \Im_{V^{-}}\right]=\mathbb{E}\left[\sum_{I \in \Lambda} 1_{\left\{\tau_{I}=V\right\}} Z_{V}^{f}(I)+1_{\left\{\tau_{I} \neq V, \forall I \in \Lambda\right\}} M_{V}^{f} \mid \Im_{V^{-}}\right] \\
& =\sum_{I \in \Lambda} Z_{V}^{f}(I) \mathbb{P}\left[V=\tau_{I} \mid \Im_{V^{-}}\right]+\mathbb{E}\left[1_{\left\{\tau_{I} \neq V, \forall I \in \Lambda\right\}} M_{V}^{f} \mid \Im_{V^{-}}\right] .
\end{aligned}
$$

Contudo, em $\left\{\Delta A_{V}(\emptyset)=0\right\}$, temos

$$
\mathbb{P}\left\{V \neq \tau_{I}, \forall I \in \Lambda \mid \Im_{V^{-}}\right\}=1-\sum_{I \in \Lambda} \Delta A_{V}(I)=\Delta A_{V}(\emptyset)=0,
$$

de maneira que $\mathbb{E}\left[1_{\left\{\tau_{I} \neq V, \forall I \in \Lambda\right\}} M_{V}^{f} \mid \Im_{V^{-}}\right]=0=Z_{V}^{f}(\emptyset) \Delta A_{V}(\emptyset)$ e

$$
M_{V-}^{f}=\sum_{I \in \Lambda} Z_{V}^{f}(I) \Delta A_{V}(I)+Z_{V}^{f}(\emptyset) \Delta_{V}(\emptyset) .
$$

Com o Lema 1.2.5, determinaremos na próxima proposição, uma forma explícita para $C^{f}(I)$.

Proposição 1.2.6: O processo $C^{f}(I), I \in \Lambda$ na representação do martingal em (1.2.3) pode ser escrito como

$$
C_{t}^{f}(I)=Z_{t}^{f}(I)-Z_{t}^{f}(\emptyset) .
$$

Prova: Usando a notação $\bar{M}_{t}=\mathbb{E}(f(\mathrm{~S}))+\sum_{I \in \Lambda} \int_{0}^{t}\left(Z_{t}^{f}(I)-Z_{t}^{f}(\emptyset)\right) d M_{s}(I)$ temos, para todo $S_{i}, 1 \leq i \leq n$ :

$$
\begin{aligned}
\Delta \bar{M}_{S_{i}} & =\sum_{I \in \Lambda} Z_{S_{i}}^{f}(I)\left[\Delta N_{S_{i}}(I)-\Delta A_{S_{i}}(I)\right]-\sum_{I \in \Lambda} Z_{S_{i}}^{f}(\emptyset)\left[\Delta N_{S_{i}}(I)-\Delta A_{S_{i}}(I)\right] \\
& =\sum_{I \in \Lambda} Z_{S_{i}}^{f}(I) \Delta N_{S_{i}}(I)-\sum_{I \in \Lambda} Z_{S_{i}}^{f}(I) \Delta A_{S_{i}}(I)-Z_{S_{i}}^{f}(\emptyset)\left[1-\sum_{I \in \Lambda} \Delta A_{S_{i}}(I)\right] \\
& =M_{S_{i}}^{f}-M_{S_{i}^{-}}^{f} \\
& =\Delta M_{S_{i}}^{f}
\end{aligned}
$$


e portanto $\Delta\left(M^{f}-\bar{M}\right)_{S_{i}}=0$ para todo $S_{i}, 1 \leq i \leq n$

Por outro lado, para qualquer tempo de parada limitado e previsível $V$, temos para $M^{*}=M^{f}-\bar{M}$,

$$
\begin{aligned}
0 & =\mathbb{E}\left(\Delta M_{V}^{*} \mid \Im_{V-}\right) \\
& =\mathbb{E}\left(\Delta M_{V}^{*} 1_{\left(\Delta M^{*} \neq 0\right)} \mid \Im_{V-}\right) \\
& =\mathbb{E}\left(\sum_{I \in \Lambda} C_{V}^{*}(I)\left[\Delta N_{V}(I)-\Delta A_{V}(I)\right] 1_{\left(\Delta M^{*} \neq 0\right)} \mid \Im_{V^{-}}\right) \\
& =\mathbb{E}\left(-\sum_{I \in \Lambda} C_{V}^{*}(I) \Delta A_{V}(I) 1_{\left(\Delta M^{*} \neq 0\right)} \mid \Im_{V^{-}}\right) \\
& =-\sum_{I \in \Lambda} C_{V}^{*}(I) \Delta A_{V}(I) P\left(\Delta M^{*} \neq 0 \mid \Im_{V^{-}}\right)
\end{aligned}
$$

onde a terceira igualdade vem do teorema da representação geral de um martingal com $C^{*}(I), I \in \Lambda$ previsível e $\Delta N_{V}(I) 1_{\left(\Delta M_{V} \neq 0\right)}=0$.

Assim, em $\left\{\mathbb{P}\left(\Delta M_{V}^{*} \neq 0 \mid \Im_{V^{-}}\right)>0\right\}$ temos que $\sum_{I \in \Lambda} C_{V}^{*}(I) \Delta A_{V}(I)=0$, o que implica que

$$
\Delta M_{V}^{*}=\sum_{I \in \Lambda} C_{V}^{*}(I)\left(\Delta N_{V}(I)-\Delta A_{V}(I)\right)=0
$$

No conjunto onde $P\left(\Delta M_{V}^{*} \neq 0 \mid \Im_{V^{-}}\right)=0$ temos imediatamente que $\Delta M_{V}^{*}=0$.

Portanto $M^{*}$ não possui saltos em qualquer tempo previsível $V$ e temos o resultado esperado.

Usando a Proposição (1.2.6) e o Lema (1.2.5), temos que: 


$$
\begin{aligned}
M_{t}^{f}= & M_{t^{-}}^{f}+\Delta M_{t}^{f} \\
= & \sum_{I \in \Lambda^{0}} Z_{t}^{f}(I) \Delta A_{t}(I)+\sum_{I \in \Lambda} Z_{t}^{f}(I)\left(\Delta N_{t}(I)-\Delta A_{t}(I)\right)- \\
& -Z_{t}^{f}(\emptyset) \sum_{I \in \Lambda}\left(\Delta N_{t}(I)-\Delta A_{t}(I)\right) \\
= & \sum_{I \in \Lambda} Z_{t}^{f}(I) \Delta N_{t}(I)+Z_{t}^{f}(\emptyset)\left(1-\sum_{I \in \Lambda} \Delta A_{t}(I)\right)-Z_{t}^{f}(\emptyset) \sum_{I \in \Lambda}\left(\Delta N_{t}(I)-\Delta A_{t}(I)\right) \\
= & \sum_{I \in \Lambda} Z_{t}^{f}(I) \Delta N_{t}(I)+Z_{t}^{f}(\emptyset)\left(1-\sum_{I \in \Lambda} \Delta N_{t}(I)\right)
\end{aligned}
$$

ou seja, $M_{t}^{f}=Z_{t}^{f}(I), I \in \Lambda$, se houver falha do conjunto $I$ em $t$ e $M_{t}^{f}=Z_{t}^{f}(\emptyset)$ se não houver falha. Assim $Z_{t}^{f}(I)$ pode ser entendida como a esperança condicional de $f(\mathrm{~S})$ dado $\Im_{t}$, se houver falha do conjunto $I$ no instante $t$.

Voltando a Proposição 1.2.6, a integral em (1.2.3) pode então ser vista como um "fator de correção" a medida que o tempo passa para a estimativa $M_{t}^{f}=\mathbb{E}\left(f(\mathrm{~S}) \mid \Im_{t}\right)$ de $f(\mathrm{~S})$. Se o integrando $C^{f}(I)$ é não positivo para todo $I \in \Lambda$ então cada falha significa uma perda para o valor estimado (ver Exemplo 1.1.1). Este tipo de comportamento nos leva a seguinte definição :

Definição 1.2.7: Dizemos que $\mathrm{S}=\left(S_{i}\right)_{1 \leq i \leq n}$ é enfraquecido por falhas (WBF) se, para toda função crescente limitada $f: \mathbb{R}_{+}^{n} \rightarrow \mathbb{R}$ e para todo conjunto $I \in \Lambda$,

$$
Z^{f}(\emptyset) \geq Z^{f}(I)
$$

ou equivalentemente

$$
Z_{\tau_{I}}^{f}(I)=\mathbb{E}\left(f(\mathbf{S}) \mid \Im_{\tau_{I}}\right) \leq Z_{\tau_{I}}^{f}(\emptyset) \quad \text { q.c. em } \quad\left\{\tau_{I}<\infty\right\}
$$

Veremos a seguir teoremas com algumas condições para associação de S.

Teorema 1.2.8: Suponhamos que $\mathrm{S}$ seja (WBF) e que todos os compensadores $A_{t}(I), I \in \Lambda$ sejam contínuos. Então $\mathrm{S}$ é associado.

Prova: Como $A_{t}(I)$ são contínuos, $\Delta A_{t}(I) \equiv 0$ para $\forall I \in \Lambda$ e $\Delta A_{s}(\emptyset)=1 \mathrm{e}$ portanto por (1.2.1) e (1.2.2) 


$$
\operatorname{Cov}\left(f(\mathbf{S}), g(\mathbf{S})=\mathbb{E}\left(\sum_{I \in \Lambda} \int_{0}^{\infty} C_{s}^{f}(I) C_{s}^{g}(I) d A_{s}(I)\right),\right.
$$

que é não negativa se $\mathrm{S}$ é WBF.

Os compensadores $A_{t}(I)$ são contínuos se e somente se todos os tempos de parada $\tau_{I}$ forem totalmente inacessíveis e esta é uma propriedade muito comum nos modelos de tempo contínuo que não envolvem tempos determinísticos, como por exemplo o Processo de Poisson.

No caso de modelos com tempos discretos, entretanto, precisaremos de um conjunto alternativo de condições para que $\operatorname{Cov}_{\Delta A}\left(C^{f}(\cdot), C^{g}(\cdot)\right)$ seja não negativa. Para tanto usaremos a seguinte definição :

Definição 1.2.9: Dizemos que $\mathrm{S}=\left(S_{i}\right)_{1 \leq i \leq n}$ é enfraquecida monotonamente por falhas se (i) S é WBF e (ii) para funções limitadas crescentes $f: \mathbb{R}_{+}^{n} \rightarrow \mathbb{R}$ e $I, J \in \Lambda$

$$
\left[Z^{f}(I)-Z^{f}(J)\right] \Delta A(I) \Delta A(J) \geq 0 \text { se } I \subset J
$$

ou seja, um aumento no tamanho do conjunto de falhas decresce estocasticamente a vida residual dos componentes restantes.

Definição 1.2.10: Consideremos a distribuição de probabilidade $\left\{A_{S}(I), I \in \Lambda^{0}\right\}$ do conjunto de falhas no ponto de salto $S$. Diremos que $\left\{A_{S}(I), I \in \Lambda^{0}\right\}$ é fracamente associado se sua covariância

$$
\sum_{I \in \Lambda^{0}} \bar{f}_{S}(I) \bar{g}_{S}(I) \Delta A_{S}(I)-\left(\sum_{I \in \Lambda^{0}} \bar{f}_{S}(I) \Delta A_{S}(I)\right)\left(\sum_{I \in \Lambda^{0}} \bar{g}_{S}(I) \Delta A_{S}(I)\right)
$$

é não negativa para quaisquer funções crescentes $\bar{f}_{S}$ e $\bar{g}_{S}$ em $\Lambda^{0}$.

Teorema 1.2.11: Suponhamos que $\mathrm{S}=\left(S_{i}\right)_{1 \leq i \leq k}$ seja enfraquecido monotonamente por falhas e que $\left\{\Delta A_{S}(I), I \in \Lambda^{0}\right\}$ seja fracamente associada para todo tempo previsível $S$. Então $\mathrm{S}$ é associado. 
Prova: Considerando que $\bar{f}_{s}(I)=-C_{s}^{f}(I)$ e que $\bar{g}_{s}(I)=-C_{s}^{g}(I)$ obtemos, pela Proposição 1.2.4, $\sum_{s \leq t} \operatorname{Cov}_{\triangle A}\left(C^{f}(\cdot), C^{g}(\cdot)\right)$ é não negativa. 


\subsection{Importância de Componentes.}

Sejam $\tau$ e $S_{i}, i=1, \ldots, n$, os tempos de vida de um sistema e de seus componentes, respectivamente. Birnbaum (1969) define ( a importância da confiabilidade da componente $i$ para a confiabilidade do sistema como

$$
I_{t}^{B}\left(\tau, S_{i}\right)=\mathbb{P}\left\{\tau>t \mid S_{i}>t\right\}-\mathbb{P}\left\{\tau>t \mid S_{i} \leq t\right\}, \quad i=1, \ldots, n
$$

Nas aplicações, esta medida contém limitações pois depende do momento fixado do tempo $t$. Para resolver tal problema, várias medidas de importância foram propostas (Barlow e Proschan (1975), Natvig (1985)), sendo que a maioria delas são integrais ponderadas de $I_{t}^{B}\left(\tau, S_{i}\right)$ sobre $t$.

Bergman (1985) observa que, na Teoria da Confiabilidade, várias medidas de importância podem ser obtidas através do estudo da troca do tempo de vida esperado do sistema, devido a diferentes variações de distribuição do tempo de vida do componente em questão. Esta noção é facilmente comprovada no caso de componentes independentes. Neste caso,

$$
\begin{aligned}
\mathbb{E}(\tau) & =\int_{0}^{\infty} \mathbb{P}(\tau>t) d t \\
& =\int_{0}^{\infty}\left[\mathbb{P}\left(\tau>t \mid S_{i}>t\right) \mathbb{P}\left(S_{i}>t\right)+\mathbb{P}\left(\tau>t \mid S_{i} \leq t\right) \mathbb{P}\left(S_{i} \leq t\right)\right] d t \\
& =\int_{0}^{\infty}\left[\mathbb{P}\left(S_{i}>t\right) I_{t}^{B}\left(\tau, S_{i}\right)+\mathbb{P}\left(\tau>t \mid S_{i} \leq t\right)\right] d t
\end{aligned}
$$

Assim, se $F_{i}$ e $G_{i}$ denotam a distribuição original e a distribuição modificada do componente $i$, respectivamente, a importância do componente $i$ com respeito a esta modificação é dada por

$$
\int_{0}^{\infty}\left(\bar{G}_{i}(t)-\bar{F}_{i}(t)\right) I_{t}^{B}\left(\tau, S_{i}\right) d t
$$

onde $\bar{F}_{i}(t)=1-F(t)$ e $\bar{G}_{i}(t)=1-G(t)$ são as funções de sobrevivência de $F$ e $G$. 
Como uma aplicação desta noção de importância, podemos obter a importância de Natvig através de um reparo mínimo do componente.

Um reparo mínimo do componente $i$ significa que, imediatamente depois de sua primeira falha, o componente é reparado para ter a mesma distribuição do tempo de vida que tinha imediatamente anterior à falha. Isto é, um tempo de vida adicional $S_{i}^{\prime}$ com função de sobrevivência condicional

$$
\mathbb{P}\left\{S_{i}^{\prime}>s \mid S_{i}=t\right\}=\mathbb{P}\left\{S_{i}>s+t \mid S_{i}>t\right\}
$$

é adicionado ao tempo de vida original $S_{i}$, resultando em um novo tempo de vida com função de sobrevivência

$$
\begin{aligned}
\bar{G}_{i}(t) & =\mathbb{P}\left\{S_{i}>t\right\}+\int_{0}^{t} \mathbb{P}\left\{S_{i}^{\prime}>t-s \mid S_{i}=s\right\} d F_{i}(s) \\
& =\bar{F}_{i}(t)-\bar{F}_{i}(t) \ln \bar{F}_{i}(t) .
\end{aligned}
$$

Portanto,

$$
\begin{aligned}
I^{N}\left(\tau, S_{i}\right) & =\int_{0}^{\infty}\left(\bar{G}_{i}(t)-\bar{F}_{i}(t) I_{t}^{B}\left(\tau, S_{i}\right) d t\right. \\
& =\int_{0}^{t}-\bar{F}_{i}(t) \ln \bar{F}_{i}(t) I_{t}^{B}\left(\tau, S_{i}\right) d t
\end{aligned}
$$

é a importância da confiabilidade do componente para a confiabilidade do sistema de Natvig (1985).

No contexto dessa dissertação, provaremos que esta medida de importância da confiabilidade do componente $i$ pode ser obtida através da modificação do martingal $\mathbb{E}\left[S \mid \Im_{t}\right]$ no tempo de falha $T_{i}, i=1, \ldots, n$. Esta abordagem é de Norros (1986a) e sua maior vantagem é que a interdependência dos tempos de vida dos componentes pode ser considerada sem maiores complicações .

Nesta seção assumiremos as seguintes hipóteses:

i) $\mathbb{P}\left(0<S_{i}<\infty\right)=1 \quad i=1, \ldots, n$,

ii) Para todo $i, j$ com $i \neq j, \mathbb{P}\left(S_{i}=S_{j}\right)=0 \mathrm{e}$ 
iii) Todos os compensadores $A_{t}(i)=A_{t}\{i\}$ são contínuos. $A_{t}(I)=0$.

Observe que, pela hipótese (ii), I contém mais que um elemento implica que

Seja $\tau$ o tempo de vida de um sistema $\operatorname{com} \mathbb{E}(\tau)<\infty$ e seja $\left(M_{t}^{\tau}\right)_{t \geq 0}$ uma versão contínua à direita com limites à esquerda do martingal uniformemente integrável $\left(\mathbb{E}\left(\tau \mid \Im_{t}\right)\right)_{t \geq 0}$. Desejamos calcular $\mathbb{E}\left[\Delta M_{S_{i}}^{\tau}\right]=\mathbb{E}\left[M_{S_{i}}^{\tau}-M_{S_{i}^{-}}^{\tau}\right]$ onde $S_{i}$ é o tempo de vida do componente $i, i=1, \ldots, n$.

Exemplo 1.3.1: Consideremos o caso de um sistema formado por apenas um componente, isto é, $\tau=S_{1}, \mathbb{E}(\tau)<\infty$ e $n=1$. Se $\Im_{t}=\sigma\left\{1_{\{\tau>s\}} s \leq t\right\}$, então

$$
M_{t}^{\tau}=\mathbb{E}[\tau \mid \tau>t] 1_{\{\tau>t\}}+\tau 1_{\{r \leq t\}} .
$$

Contudo,

$$
\begin{aligned}
\mathbb{E}[\tau \mid \tau>t] & =\int_{0}^{\infty} \mathbb{P}\{\tau>v \mid \tau>t\} d v \\
& =\int_{0}^{t} \mathbb{P}\{\tau>v \mid \tau>t\} d v+\int_{t}^{\infty} \mathbb{P}\{\tau>v \mid \tau>t\} d v \\
& =t+\int_{t}^{\infty} \frac{\mathbb{P}(\tau>v)}{\mathbb{P}(\tau>t)} d v \\
& =t+\frac{1}{\bar{F}(t)} \int_{0}^{\infty} \bar{F}(u+t) d u
\end{aligned}
$$

e concluímos que

$$
M_{t}^{\tau}=\tau \wedge t+\frac{1}{\bar{F}(t)} \int_{0}^{\infty} \bar{F}(u+t) d u 1_{\{\tau>t\}} .
$$

Assim, $\Delta M_{\tau}^{\tau}=-\frac{1}{\bar{F}(\tau)} \int_{0}^{\infty} \bar{F}(\tau+u) d u \quad \mathrm{e}$ 


$$
\begin{aligned}
\mathbb{E}\left[\Delta M_{\tau}^{\tau}\right] & =\mathbb{E}\left[-\frac{1}{\bar{F}(\tau)} \int_{0}^{\infty} \bar{F}(\tau+u) d u\right] \\
& =\int_{0}^{\infty}\left(-\frac{1}{\bar{F}(s)} \int_{0}^{\infty} \bar{F}(s+u) d u\right) d F(s) \\
& =\int_{0}^{\infty}-\frac{1}{\bar{F}(s)}\left(\int_{s}^{\infty} \bar{F}(v) d v\right) d F(s) \\
& \left.=\int_{0}^{\infty}-\bar{F}(v)\left(\int_{0}^{v} \frac{d F(s)}{\bar{F}(s)}\right) d v\right) \\
& =-\int_{0}^{\infty} \bar{F}(v) \ln \bar{F}(v) d v \\
& =-\int_{0}^{\infty} \bar{F}(v) \ln \bar{F}(v) I_{v}(\tau, \tau) d v \\
& =I^{N}(\tau, \tau) .
\end{aligned}
$$

De uma maneira geral, o problema é colocado na proposição a seguir:

Proposição 1.3.2: $\operatorname{Sejam~}(\Omega, \Im, P)$ um espaço de probabilidade e $\left(\mathcal{G}_{t}\right)_{t \geq 0}$ uma família de sub $\sigma$-álgebras de $\Im$, crescentes, contínuas à direita e com $\mathcal{G}_{0}$ contendo todos os conjuntos de $P$-medida nula. Sejam $S$ e $T\left(\mathcal{G}_{t}\right)$-tempos de paradas, $N_{t}=1_{\{T \leq t\}}$ e $\left(A_{t}\right)_{t \geq 0}$ o $\mathcal{G}_{t}$-compensador de $\left(N_{t}\right)_{t \geq 0}$. Suponhamos que $\mathbb{E}(S)<\infty$, que $\left(A_{t}\right)_{t \geq 0}$ seja contínuo e $M_{t}^{S}$ uma versão contínua à direita, com limites à esquerda do martingal uniformemente integrável $\mathbb{E}\left(S \mid \mathcal{G}_{t}\right)$. Então

$$
\mathbb{E}\left(\Delta M_{T}^{S}\right)=-\mathbb{E}\left(S A_{T}\right)+E(S)=-\operatorname{Cov}\left(S, A_{T}\right)
$$

Prova: Notemos que $\mathbb{E}\left(M_{T}^{S}\right)=\mathbb{E}\left\{\mathbb{E}\left(S \mid \mathcal{G}_{T}\right)\right\}=E(S)$ e que $\left(N_{t}-A_{t}\right)_{t \geq 0}$ é um martingal com $\mathbb{E}\left(N_{t}-A_{t}\right)=\mathbb{E}\left(N_{0}-A_{0}\right)=0$, assim $\mathbb{E}\left(A_{T}\right)=\mathbb{E}\left(N_{T}\right)=1$.

Por outro lado, 


$$
\begin{aligned}
\mathbb{E}\left[M_{T^{-}}^{S}\right] & =\mathbb{E}\left\{\int_{0}^{\infty} M_{t^{-}}^{S} d N_{t}\right\} \\
& =\mathbb{E}\left\{\int_{0}^{\infty} M_{t^{-}}^{S} d A_{t}\right\} \\
& =\mathbb{E}\left\{\int_{0}^{\infty} M_{t}^{S} d A_{t}\right\} \\
& =\mathbb{E}\left\{M_{T}^{S} A_{T}\right\}
\end{aligned}
$$

A segunda igualdade acima segue do fato de que $\left(N_{t}-A_{t}\right)_{t \geq 0}$ é um martingal e de que $M_{t^{-}}^{S}$ é previsível e portanto $\left(\int_{0}^{s} M_{t^{-}}^{S} d\left(N_{t}-A_{t}\right)\right)_{s \geq 0}$ é um martingal com média 0 . A terceira igualdade ocorre porque $A_{t}$ é um processo natural crescente e, finalmente, a última igualdade segue da fórmula integral de Dellacherie.

Concluímos então que

$$
\begin{aligned}
\mathbb{E}\left[\Delta M_{T}^{S}\right] & =\mathbb{E}\left[M_{T}^{S}\right]-\mathbb{E}\left[M_{T^{-}}^{S}\right] \\
& =\mathbb{E}[S]-\mathbb{E}\left[A_{T} \mathbb{E}\left(S \mid \mathcal{G}_{T}\right)\right] \\
& =\mathbb{E}[S]-\mathbb{E}\left[\mathbb{E}\left(A_{T} S \mid \mathcal{G}_{T}\right)\right] \\
& =\mathbb{E}[S] \mathbb{E}\left[A_{T}\right]-\mathbb{E}\left[A_{T} S\right] \\
& =-\operatorname{Cov}\left(A_{T}, S\right) .
\end{aligned}
$$

No contexto de sistemas coerentes e de seus componentes, consideremos o tempo de vida do sistema $\tau$ com $\mathbb{E}\left[\tau^{2}\right] \leq \infty$, uma condição que garante a existência de $\mathbb{E}\left[\Delta M_{S_{i}}^{\tau}\right]$.

Definição 1.3.3: Com as hipóteses (1.3.1) e supondo que $\mathbb{E}\left[\tau^{2}\right]<\infty$, definimos:

i) A importância absoluta do componente $i$ para o sistema $\tau$ como

$$
I_{A}\left(\tau, S_{i}\right)=-\mathbb{E}\left[\Delta M_{S_{i}}^{\tau}\right]=\operatorname{Cov}\left(\tau, A_{S_{i}}(i)\right)
$$

ii) A importância relativa do componente $i$ para o sistema $\tau$ como 


$$
I_{R}\left(\tau, S_{i}\right)=\frac{I_{A}\left(\tau, S_{i}\right)}{\sum_{i=i}^{n} I_{A}\left(\tau, T_{i}\right)} .
$$

Podemos provar que as variáveis aleatórias $A_{S_{i}}(i), i=1, \ldots, n$, são independentes e identicamente distribuídas com distribuição exponencial de parâmetro 1.

Lema 1.3.4: Sob as hipóteses (1.3.1) $A_{\infty}(i)=A_{S_{i}}(i), i=1, \ldots, n$, são independentes e exponencialmente distribuídas com parâmetro 1.

Prova: É suficiente mostrar que, para quaisquer números reais $u_{1}, u_{2}, \ldots, u_{n}$,

$$
\mathbb{E}\left\{\exp \left[\sum_{j=1}^{n} i u_{j} A_{\infty}(j)\right]\right\}=\prod_{j=1}^{n} \frac{1}{1-i u_{j}} .
$$

Observe que

$$
\begin{aligned}
m_{t}(j) & =\left(1-i u_{j}\right)^{N_{t}(j)} \exp \left[i u_{j} A_{t}(j)\right] \\
& =1+\int_{0}^{t} i u_{j} \exp \left[i u_{j} A_{s}(j)\right] d\left\{A_{s}(j)-N_{s}(j)\right\}
\end{aligned}
$$

pois, se $t<S_{j}$,

$$
\begin{aligned}
1+\int_{0}^{t} i u_{j} \exp \left[i u_{j} A_{s}(j)\right] d\left\{A_{s}(j)-N_{s}(j)\right\} & =1+\int_{0}^{t} i u_{j} \exp \left[i u_{j} A_{s}(j)\right] d A_{s}(j) \\
& =\exp \left[i u_{j} A_{t}(j)\right] \\
& =\left(1-i u_{j}\right)^{N_{t}(j)} \exp \left[i u_{j} A_{t}(j)\right]
\end{aligned}
$$

e se $t \geq S_{j}$, 


$$
\begin{aligned}
1+\int_{0}^{t} i u_{j} \exp \left[i u_{j} A_{s}(j)\right] d\left\{A_{s}(j)-N_{s}(j)\right\}= & +\int_{0}^{S_{j}} i u_{j} \exp \left[i u_{j} A_{s}(j)\right] d A_{s}(j)- \\
& -\int_{0}^{t} i u_{j} \exp \left[i u_{j} A_{s}(j)\right] d N_{s}(j) \\
= & 1+\exp \left[i u_{j} A_{S_{j}}(j)\right]-1-i u_{j} \exp \left[i u_{j} A_{S_{j}}(j)\right] \\
= & \left(1-i u_{j}\right)^{N_{t}(j)} \exp \left[i u_{j} A_{t}(j)\right] .
\end{aligned}
$$

Contudo, como $\left(A_{t}-N_{t}\right)_{t \geq 0}$ é um martingal e $i u_{j}\left[\exp \left[i u_{j} A_{t}(j)\right]\right.$ é previsivel, podemos concluir que $m_{t}(j)$ é um martingal e utilizando a regra de diferenciação de Stieltjes, temos

$$
m_{t}(i) m_{t}(j)-1=\int_{0}^{t} m_{s^{-}}(i) d m_{s}(j)+\int_{0}^{t} m_{s^{-}}(j) d m_{s}(i)+\sum_{s \leq t} \Delta m_{s}(i) \Delta m_{s}(j) .
$$

Como os $A_{t}(i)$ 's são contínuos e $\mathbb{P}\left(S_{i}=S_{j}\right)=0$ por hipótese, concluímos que $\sum_{s \leq t} \Delta m_{s}(i) \Delta m_{s}(j)=0$ e que $m_{t}(i) m_{t}(j)$ é um martingal, com $<m(i), m(j)>_{t}=0$. Isto é, os $\left(m_{t}(i)\right)_{t \geq 0}$ são martingais ortogonais. Assim, seu produto é um martingal limitado e obtemos

$$
\mathbb{E}\left\{\prod_{j=1}^{n}\left(1-i u_{j}\right) \exp \left[i u_{j} A_{T_{j}}(j)\right]\right\}=\mathbb{E}\left[\prod_{j=1}^{n} m_{0}(j)\right]=\mathbb{E}[1]=1
$$

Logo

$$
\mathbb{E}\left\{\exp \left[\sum_{j=1}^{n} i u_{j} A_{T_{j}}(j)\right]\right\}=\prod_{j=1}^{n} \frac{1}{1-i u_{j}}
$$

Portanto os tempos de vida $S_{i}$ são relacionados com as variáveis aleatórias $A_{S_{i}}(i)$, os valores finais de seus compensadores, que são independentes com distribuição exponencial de parâmetro 1. Norros (1986b) prova que essa correspondência é bijetora. Mais precisamente, dados valores exponenciais $X$ 's, os valores $S_{i}$ podem ser gerados como segue: 
Os compensadores $A_{t}(i)$ são determinísticos entre os tempos $T_{k}, k \geq 0$. A cada falha $T_{k}$, novas funções determinística $a_{t}(i)$ são escolhidas (dependendo dos valores de $\left.\left\{T_{k}, X_{k}, 1 \leq k \leq n\right\}\right)$ tais que $A_{T_{k}+t}(i)=A_{T_{k}}(i)+a_{t}(i)$ para $t \leq T_{k+1}-T_{k}$.

Para gerar os tempos de vida $S_{i}$, basta observar os compensadores, até que algum deles, digamos $A_{t}(i)$, alcance um pré-determinado valor exponencial $X$ em algum tempo $t$. Então $S_{i}$ assume o valor $t$ e o algoritmo continua com a troca correspondente nos compensadores.

No caso de componentes independentes, temos que

$$
A_{S_{i}}(i)=-\ln \bar{F}_{i}\left(S_{i}\right)
$$

onde $\bar{F}_{i}$ é a função de sobrevivência do componente $i$. Dessa maneira, a importância absoluta do componente $i$ é dado por:

$$
I_{A}\left(\tau, S_{i}\right)=-\operatorname{Cov}\left(\tau, \ln \bar{F}_{i}\left(S_{i}\right)\right)
$$

e neste caso, a definição acima é equivalente a de Natvig, como demonstraremos na seguinte proposição :

Proposição 1.3.5: No caso de componentes independentes,

$$
I_{A}\left(\tau, S_{i}\right)=-\int_{0}^{\infty} \bar{F}_{i}(t) \ln \bar{F}_{i}(t) I_{t}^{B}\left(\tau, S_{i}\right) d t
$$

Prova: Como existe uma correspondência biunívoca entre $S_{i}$ e $A_{S_{i}}(i)$, podemos usar a condicional em $S_{i}$ e obter 


$$
\begin{aligned}
\mathbb{E}\left[\tau A_{S_{i}}(i)\right]= & \mathbb{E}\left\{\mathbb{E}\left[\tau A_{S_{i}}(i) \mid A_{S_{i}}(i)\right]\right\} \\
= & \mathbb{E}\left\{A_{S_{i}}(i) \mathbb{E}\left[\tau \mid A_{S_{i}}(i)\right]\right\} \\
= & \mathbb{E}\left\{A_{S_{i}}(i) \int_{0}^{\infty} \mathbb{P}\left(\tau>t \mid A_{S_{i}}(i)\right) d t\right\} \\
= & \mathbb{E}\left\{A_{S_{i}}(i) \int_{0}^{\infty} \mathbb{P}\left(\tau>t \mid S_{i}\right) d t\right\} \\
= & \mathbb{E}\left\{A_{S_{i}}(i) \int_{0}^{\infty}\left[1_{\left\{S_{i} \leq t\right\}} \mathbb{P}\left(\tau>t \mid S_{i} \leq t\right)+1_{\left\{S_{i}>t\right\}} \mathbb{P}\left(\tau>t \mid S_{i}>t\right)\right] d t\right\} \\
= & \int_{0}^{\infty}\left[\mathbb{P}\left(\tau>t \mid S_{i} \leq t\right) \mathbb{E}\left(A_{S_{i}}(i) 1_{\left\{S_{i} \leq t\right\}}\right)+\right. \\
& \left.+\mathbb{P}\left(\tau>t \mid S_{i}>t\right) \mathbb{E}\left(A_{S_{i}}(i) 1_{\left\{S_{i}>t\right\}}\right)\right] d t
\end{aligned}
$$

A última igualdade acima consegue-se pelo teorema de Fubini. Contudo,

$$
\begin{aligned}
\mathbb{E}\left(A_{S_{i}}(i) 1_{\left\{S_{i} \leq t\right\}}\right) & =\mathbb{E}\left(-\ln \bar{F}_{i}\left(S_{i}\right) 1_{\left\{S_{i} \leq t\right\}}\right) \\
& =\int_{0}^{-\ln \bar{F}_{i}(t)} x e^{-x} d x \\
& =\ln \bar{F}_{i}(t) \bar{F}_{i}(t)+\bar{F}_{i}(t) e \\
\mathbb{E}\left(A_{S_{i}}(i) 1_{\left\{S_{i}>t\right\}}\right) & =\bar{F}_{i}(t)\left(1-\ln \bar{F}_{i}(t)\right) .
\end{aligned}
$$

Substituindo esses resultados na expressão de $\mathbb{E}\left[\tau A_{S_{i}}(i)\right]$, obtemos

$$
\begin{aligned}
\mathbb{E}\left[\tau A_{S_{i}}(i)\right]= & \int_{0}^{\infty}\left[\mathbb{P}\left(\tau>t \mid S_{i} \leq t\right)\left(\ln \bar{F}_{i}(t) \bar{F}_{i}(t)+\bar{F}_{i}(t)\right)+\right. \\
& \left.+\mathbb{P}\left(\tau>t \mid S_{i}>t\right)\left(\bar{F}_{i}(t)-\bar{F}_{i}(t) \ln \bar{F}_{i}(t)\right)\right] d t
\end{aligned}
$$

Por outro lado,

$$
\begin{aligned}
\mathbb{E}(\tau) \mathbb{E}\left(A_{S_{i}}(i)\right) & =\mathbb{E}(\tau) \\
& =\int_{0}^{\infty} \mathbb{P}(\tau>t) d t \\
& =\int_{0}^{\infty}\left[\mathbb{P}\left(\tau>t \mid S_{i} \leq t\right) F_{i}(t)+\mathbb{P}\left(\tau>t \mid S_{i}>t\right) \bar{F}_{i}(t)\right] d t \\
& -28-
\end{aligned}
$$


e portanto

$$
\begin{aligned}
\operatorname{Cov}\left(\tau, A_{S_{i}(i)}\right) & =\mathbb{E}\left[\tau A_{S_{i}}(i)\right]-\mathbb{E}(\tau) \mathbb{E}\left[A_{S_{i}}(i)\right] \\
& =-\int_{0}^{\infty} \bar{F}_{i}(t) \ln \bar{F}_{i}(t)\left[\mathbb{P}\left(\tau>t \mid S_{i}>t\right)-\mathbb{P}\left(\tau>t \mid S_{i} \leq t\right)\right] d t \\
& =-\int_{0}^{\infty} \bar{F}_{i}(t) \ln \bar{F}_{i}(t) I_{t}^{B}\left(\tau, S_{i}\right) d t \\
& =I^{N}\left(\tau, S_{i}\right) .
\end{aligned}
$$

Ainda no caso de componentes independentes, seja $Q_{i}$ a distribuição conjunta do vetor $\mathrm{S}=\left(S_{1}, \ldots, S_{n}\right)$ onde a função de distribuição $F_{i}$ é modificada através de um reparo mínimo, isto é, $\bar{F}_{i}(t)$ é substituída por $\bar{F}_{i}(t)\left(1-\ln \bar{F}_{i}(t)\right)$, enquanto que as outras distribuições permanecem inalteradas. É fácil concluir, usando a Proposição 1.3.5, que

$$
I_{A}\left(\tau, S_{i}\right)=\mathbb{E}_{Q_{i}}(\tau)-\mathbb{E}_{P}(\tau)
$$

é o crescimento do tempo de vida médio do sistema causado pelo reparo mínimo do componente $i$.

No que segue, mostraremos que essa interpretação também é válida no caso geral.

Considerando as hipóteses (3.1.1.), temos

$$
I_{A}\left(\tau, S_{i}\right)=\mathbb{E}_{P}\left(\tau A_{S_{i}}(i)\right)-\mathbb{E}_{P}(\tau) \mathbb{E}_{P}\left(A_{S_{i}}(i)\right)
$$

Desde que $\mathbb{E}\left(A_{S_{i}}(i)\right)=1$, podemos considerar a medida

$$
Q_{i}(0, t]=\int_{0}^{t} A_{S_{i}}(i) d P
$$

com a derivada de Radon-Nikodym $\frac{\partial Q_{i}}{\partial P}=A_{S_{i}}(i)$ e

$$
\begin{aligned}
I_{A}\left(\tau, S_{i}\right)= & \mathbb{E}_{Q_{i}}(\tau)-\mathbb{E}_{P}(\tau) \\
& -29-
\end{aligned}
$$


Temos que mostrar que $Q_{i}$ é a medida obtida de $P$ através de um reparo mínimo. Jacod (1975) demonstra que os compensadores determinam completamente a medida de probabilidade $P$ de um processo pontual e vice-versa. Devemos assim encontrar uma formulação adequada para o reparo mínimo através dos compensadores e utilizar essa correspondência.

Para provar o resultado, recordemos que o reparo mínimo do componente $i$ significa que imediatamente depois de sua falha, o sistema como um todo, retorna ao estado imediatamente anterior à falha daquele componente. Assim, o efeito da falha do componente $i$, incluindo os efeitos nas distribuições dos tempos de vida dos outros componentes é totalmente ignorado.

Em termos dos compensadores, interpretamos que $S_{i}$ é o tempo em que a função crescente $A_{t}(i)$ atinge um valor exponencial $X$ (de parâmetro (1)). Pela propriedade da falta de memória da distribuição Exponencial, podemos ignorar a primeira falha do componente $i$ trocando a distribuição Exponencialde parâmetro (1) por uma distribuição Gama de parâmetros $(2,1)$. Enfim, a distribuição Gama $(2,1)$ é a soma de duas variáveis aleatórias independentes com distribuições Exponenciais(1), que podem ser pensadas como os valores determinando a primeira falha ignorada e o tempo de vida adicional devido ao reparo mínimo.

Seja $R(t)$ a função de risco da distribuição Gama de parâmetros $(2,1)$, isto é, $R(t)=-\ln \bar{F}(t)=t-\ln (1+t)$. Procuramos o novo compensador $B_{t}(i)$ que atinja um tempo exponencial em $S_{i}+S_{i}^{\prime}$. Agora, $A_{i}(t)=X$ é equivalente a $R\left(A_{i}(t)\right)=R(X)$ e se $X$ tem distribuição Gama de parâmetros $(2,1), R(X)$ é exponencial de parâmetro 1 e o compensador procurado é

$$
B_{t}(i)=R\left(A_{i}(t)\right)=A_{i}(t)-\ln \left(1+A_{t}(i)\right)
$$

Devemos provar então que a distribuição $Q_{i}$ obtida acima é caracterizada pela seguinte transformação nos compensadores

$$
\begin{gathered}
B_{t}(i)=A_{t}(i)-\ln \left(1+A_{t}(i)\right) \epsilon \\
B_{t}(j)=A_{t}(j) \quad \text { se } j \neq i .
\end{gathered}
$$

Concluímos portanto que $Q_{i}$ é obtida através de um reparo mínimo do componente $i$. 
Proposição 1.3.6: Nas suposições acima e se

$$
\begin{aligned}
& L_{t}=\left(\frac{\partial Q_{i}}{\partial P}\right)_{\Im_{t}}=\mathbb{E}\left(A_{S_{i}}(i) \mid \Im_{t}\right)=1-M_{t}(i)=1-N_{t}(i)+A_{t}(i), \\
& A_{t}^{\prime}(i)=A_{t}(i)-\ln \left(1+A_{t}(i)\right) \mathrm{e} \\
& A_{t}^{\prime}(j)=A_{t}(j) \quad \text { se } j \neq i . \\
& \text { o } Q_{i} \text {-compensador de } N_{t}(i) \text { é } A_{t}^{\prime}(i), i=1, \ldots, n .
\end{aligned}
$$

Prova: Usando o Lema de Komatsu, é suficiente provar que, para todo tempo de parada limitado $T$, temos:

$$
\begin{gathered}
\mathbb{E}_{Q_{i}}\left(A_{T}^{\prime}(i)\right)=\mathbb{E}_{Q_{i}}\left(N_{T}(i)\right) \quad e \\
\mathbb{E}_{Q_{i}}\left(A_{T}^{\prime}(j)\right)=\mathbb{E}_{Q_{i}}\left(N_{T}(j)\right) \text { se } j \neq i .
\end{gathered}
$$

Pela fórmula de integração de Dellacherie, temos

$$
\begin{aligned}
\mathbb{E}_{Q_{i}}\left(A_{T}^{\prime}(i)\right) & =\mathbb{E}\left[L_{T}\left(A_{T}(i)-\ln \left(A_{T}(i)+1\right)\right)\right] \\
& =\mathbb{E} \int_{0}^{T} L_{s} d A_{s}(i)-\mathbb{E} \int_{0}^{T} L_{s} \frac{1}{1+A_{s}(i)} d A_{s}(i) \\
& =\mathbb{E} \int_{0}^{T} L_{s} \frac{A_{s}(i)}{1+A_{s}(i)} d A_{s}(i) \\
& =\mathbb{E} \int_{0}^{T} L_{s^{-}} \frac{A_{s}(i)}{1+A_{s}(i)} d A_{s}(i) \\
& =\mathbb{E} \int_{0}^{T} L_{s^{-}} \frac{A_{s}(i)}{1+A_{s}(i)} d N_{s}(i) \\
& =\mathbb{E}\left[N_{T}(i) L_{S_{i}^{-}} \frac{A_{S_{i}}}{1+A_{S_{i}}}\right] \\
& =\mathbb{E}\left[N_{T}(i)\left(1-N_{S_{i}^{-}}(i)+A_{S_{i}}(i)\right) \frac{A_{S_{i}}}{1+A_{S_{i}}}\right] \\
& =\mathbb{E}\left[N_{T}(i) A_{S_{i}}\right] \\
& =\mathbb{E} Q_{Q_{i}}\left(N_{T}(i)\right) .
\end{aligned}
$$

A segunda igualdade acima provém da fórmula de integração de Dellacherie, a quarta igualdade vale porque $A_{s}$ é natural e $L_{s}$ é um martingal contínuo à direita e com 
limites à esquerda e a quinta igualdade é baseada no fato de que $\left(N_{t}-A_{t}\right)_{t \geq 0}$ é um martingal.

$$
\begin{aligned}
\mathbb{E}\left[A_{T}^{\prime}(j)\right] & =\mathbb{E} \int_{0}^{T} L_{s} d A_{s}(j)=\mathbb{E} \int_{0}^{T} L_{s^{-}} d A_{s}(j) \\
& =\mathbb{E} \int_{0}^{T} L_{s^{-}} d N_{s}(j)=\mathbb{E}\left[N_{T}(j) L_{S_{j}}^{-}\right] \\
& =\mathbb{E}\left[N_{T}(j) L_{S_{j}}\right]=\mathbb{E}_{Q_{i}}\left(N_{T}(j)\right)
\end{aligned}
$$

A penúltima igualdade segue do fato de que $\Delta L_{S_{j}}=0$ e $N_{T}(j)$ é $\Im_{S_{j}}$-mensurável. 


\section{CAPÍTULO 2}

CAPÍTULO 2: O PROCESSO DE RISCO DOS COMPONENTES E DO SISTEMA 


\section{1: A Função de Risco através do Compensador.}

Seja $\tau$ o tempo de vida de um objeto, $F(t)=P\{\tau \leq t\}$ sua função de distribuição e $\bar{F}(t)=1-F(t)$ a correspondente função de sobrevivência. Considere a função definida pela integral de Stieltjes:

$$
R(t)=\int_{(0, t]} \frac{F(d s)}{F[s, \infty)}
$$

onde $F[s, \infty)=P\{\tau \geq s\}$.

Vejamos algumas propriedades de $R(t)$ :

$1-R(0)=0$ e $R(t)$ é uma função não decrescente, pois o integrando é positivo.

$2-R(t)$ é contínua à direita, pois considerando uma sequência $\left(t_{n}\right)_{n \geq 1} \operatorname{com} t_{n} \downarrow t$ temos que

$$
(0, t]=\bigcap_{n=1}^{\infty}\left(0, t_{n}\right] \quad e \quad \int_{(0, t]} \frac{F(d s)}{F[s, \infty)}=\lim _{n \rightarrow \infty} \int_{\left(0, t_{n}\right]} \frac{F(d s)}{F[s, \infty)}
$$

3 - $R(t)$ tem os mesmos pontos de descontinuidade de $F$, com

$$
\Delta R(t)=R(t)-R\left(t^{-}\right)=\frac{P\{\tau=t\}}{F[t, \infty)} \leq 1
$$

4- No caso em que $\Delta R(t)=1$, para algum $t$, temos $P(\tau>t)=0$ e $R(s)=R(t)$, para $s \geq t$.

Como mostra o teorema abaixo, a classe das funções com as propriedades acima caracteriza as medidas de probabilidade em $(0, \infty)$.

Teorema 2.1.1: Seja $\mathcal{A}$ a classe de todas as funções $R(t)$ que são não decrescentes, contínuas à direita, $\operatorname{com} R(0)=0, \Delta R(t) \leq 1$ e $R(s)=R(t)$, para $s \geq t$ quando $\Delta R(t)=1$. Então, as fórmulas 


$$
\begin{gathered}
F(0, t]=1-e^{-R_{c}(t)} \prod_{s \leq t}(1-\Delta R(s)) e \\
R(t)=\int_{(0, t]} \frac{F(d s)}{F[s, \infty)}
\end{gathered}
$$

definem uma correspondência biunívoca entre as classes de todas as medidas de probabilidade em $(0, \infty)$ e a classe $\mathcal{A}$.

Prova: Para cada medida $F(0, t]$ podemos definir $R(t)=\int_{(0, t]} \frac{F(d s)}{F[s, \infty)}$ e inversamente, para cada $R(t)$ em $\mathcal{A}$, podemos definir a medida $F(0, t]=1-e^{R_{c}(t)} \prod_{s \leq t}(1-\Delta R(s))$. Suponhamos que existam duas medidas de probabilidades $F_{1}(0, t]$ e $F_{2}(0, t]$ tais que

$$
R(t)=\int_{(0, t]} \frac{F_{1}(d s)}{F_{1}[s, \infty)}=\int_{(0, t]} \frac{F_{2}(d s)}{F_{2}[s, \infty)} .
$$

Diferenciando as equações em $t$ e integrando convenientemente, temos:

$$
\begin{gathered}
1-\int_{0}^{t} F_{1}[s, \infty) R(d s)=F_{1}[t, \infty) e \\
1-\int_{0}^{t} F_{2}[s, \infty) R(d s)=F_{2}[t, \infty),
\end{gathered}
$$

$\operatorname{assim} F_{1}$ e $F_{2}$ satisfazem a equação $1-\int_{0}^{t} F[s, \infty) R(d s)=F[t, \infty)$ que pelo Lema 18.8 Liptser \& Shiryayev (1977b) tem solução única e igual a

$$
F_{1}[t, \infty)=F_{2}[t, \infty)=e^{-R_{c}(t)} \prod_{s \leq t}(1-\Delta R(s))
$$

Logo a correspondência é bijetora e o teorema está demonstrado.

No caso em que a função de distribuição de $\tau, F(t)=F(0, t]$ é contínua, essa relação de equivalência reduz-se a

$$
\begin{aligned}
R(t) & =-\log \bar{F}(t) \\
& -35-
\end{aligned}
$$


Nesta forma, $R(t)$ é frequentemente utilizada para caracterizar classes de distribuições de vida úteis em Teoria da Confiabilidade, como por exemplo:

a) Uma função de distribuição $F$ pertence à classe de distribuição de vida com taxa de falha crescente (IFR) se e somente se $R(t)$ é convexa.

b) Uma função de distribuição $F$ pertence à classe das distribuições NBU (New Better than Used) se e somente se $R(t+s) \geq R(t)+R(s)$, para todo $s$ e $t \geq 0$.

Com essas caracterizações, $R(t)=-\log \bar{F}(t)$ é frequentemente definida como função de risco e isso não corresponde à realidade pois, no caso geral, a distribuição pode ter uma parte discreta

$$
F(0, t]=1-e^{-R_{c}(t)} \prod_{s \leq t}(1-\Delta R(s)) .
$$

Reservando-nos contra esse inconveniente, adotaremos a seguinte definição :

Definição 2.1.2: Seja $\tau$ o tempo de vida de um objeto com função de distribuição $F$. Então

$$
R(t)=\int_{(0, t]} \frac{F(d s)}{F[s, \infty)}
$$

é denominada função de risco de $\tau$ (ou de $F$ ).

Com a definição acima preservamos a noção intuitiva de uma função de risco, ou seja,

$$
R(d t)=\frac{F(d t)}{F[t, \infty)}=P\{\tau \in d t \mid \tau \geq t\}
$$

No contexto dessa dissertação, consideraremos o indicador da falha do objeto em estudo, $N_{t}=1_{\{\tau \leq t\}}$ e a família de $\sigma$-álgebras $\left(\Im_{t}\right)_{t \geq 0}, \Im_{t}=\sigma\left\{1_{\{\tau>s\}}, s \leq t\right\}$, crescentes e contínuas à direita, completadas com os conjuntos de medida nula. Claramente, $\left(N_{t}\right)_{t \geq 0}$ é um supér-martingal e podemos concluir, utilizando a decomposição de Doob-Meyer, que existe um martingal $\left(M_{t}\right)_{t \geq 0}$ e um único processo natural crescente $\left(A_{t}\right)_{t \geq 0}(\operatorname{com} A(0)=0)$, o $\Im_{t}$-compensador de $\left(N_{t}\right)_{t \geq 0}$, tal que 


$$
N_{t}=M_{t}+A_{t}, \quad t \geq 0
$$

Podemos caracterizar o compensador $A(t)$, através da seguinte proposição :

Proposição 2.1.3: Sob as condições acima,

$$
A_{t}=\int_{(0, t \wedge \tau]} \frac{F(d s)}{F[s, \infty)} .
$$

Prova: Seja $S$ um $\left(\Im_{t}\right)$-tempo de parada.

$$
\begin{aligned}
\left.\mathbb{E}\left[A_{S}\right)\right] & =\mathbb{E}\left(\int_{(0, S \wedge \tau]} \frac{F(d u)}{F[u, \infty)}\right) \\
& =\int_{0}^{\infty}\left(\int_{(0, S \wedge t]} \frac{F(d u)}{F[u, \infty)}\right) F(d t) \\
& =\int_{0}^{S}\left(\int_{(0, t]} \frac{F(d u)}{F[u, \infty)}\right) F(d t)+\int_{S}^{\infty}\left(\int_{(0, S]} \frac{F(d u)}{F[u, \infty)}\right) F(d t) \\
& =\int_{0}^{S}\left(\int_{(0, t]} \frac{F(d u)}{F[u, \infty)}\right) F(d t)+[1-F(S)] \int_{(0, S]} \frac{F(d u)}{F[u, \infty)}
\end{aligned}
$$

Integrando por partes, temos que

$$
\begin{aligned}
\int_{0}^{S}\left(\int_{(0, t]} \frac{F(d u)}{F[u, \infty)}\right) F(d t) & =F(S) \int_{(0, S]} \frac{F(d u)}{F[u, \infty)}-\int_{(0, S]} \frac{F\left(u^{-}\right) F(d u)}{F[u, \infty)} \\
& =[F(S)-1] \int_{(0, S]} \frac{F(d u)}{F[u, \infty)}+F(S)
\end{aligned}
$$

e portanto obtemos que

$$
E\left[A_{S}\right]=F(S)=E\left\{1_{\{\tau \leq S\}}\right\}=E\left[N_{S}\right]
$$

Usando o Lema de Komatsu, provamos que $\left(N_{t}-A_{t}\right)_{t \geq 0}$ é um martingal. Desde que $A_{0}=0$ e utilizando a unicidade da decomposição de Doob-Meyer, concluímos que $\left(A_{t}\right)_{t \geq 0}$ é o $\Im_{t}$-compensador de $\left(N_{t}\right)_{t \geq 0}$. 
O fato importante a ser considerado é que o $\left(\Im_{t}\right)$-compensador

$$
A_{t}=\int_{(0, t \wedge \tau]} \frac{F(d s)}{F[s, \infty)}
$$

é a nossa função de risco no conjunto de interesse $\{t \leq \tau\}$, a qual caracteriza as distribuições IFR e NBU no caso contínuo e univariado.

Para generalizar a noção de risco através dos compensadores, notemos primeiramente que estes são determinados relativamente a uma família de $\sigma$-álgebras $\left(\Im_{t}\right)_{t \geq 0}$ fixada. Se mudarmos esta família mudaremos o compensador. Portanto, ao caracterizar uma distribuição através dos compensadores devemos considerar a família de $\sigma$-álgebras envolvida. Esta generalização de classes de distribuição de vida baseadas em noções de ordenação estocástica condicional à família de $\sigma$-álgebras $\left(\Im_{t}\right)_{t \geq 0}$ é apresentada em Arjas (1981). 


\subsection{Classes de Distribuições $\operatorname{MIFR} \mid\left(\Im_{t}\right)$.}

Consideremos um vetor aleatório $\mathrm{S}=\left(S_{1}, \ldots, S_{n}\right), S_{i}>0, i=1, \ldots, n$, provavelmente representando os tempos de vida dos componentes de um sistema.

Seja $\theta_{t}\left(S_{i}\right)=\left(S_{i}-t\right)^{+}=\max \left(S_{i}-t, 0\right)$, o tempo de vida residual de $S_{i}$ no instante t. Denotaremos $\theta_{t} \mathrm{~S}=\left(\theta_{t} S_{1}, \ldots, \theta_{t} S_{n}\right)$.

Começaremos por definir a classe univariada de distribuições de vida IFR relativa a uma família $\left(\Im_{t}\right)_{t \geq 0}$.

Definição 2.2.1: Dizemos que o tempo de vida $\tau$ (ou sua distribuição ) é IFR|( $\left.\Im_{t}\right)$ se, para todo $0 \leq t^{*} \leq t$ e $s \in \mathbb{R}$,

$$
\mathbb{P}\left\{\theta_{t} \tau>s \mid \Im_{t}\right\} \leq \mathbb{P}\left\{\theta_{t^{*}} \tau>s \mid \Im_{t^{*}}\right\} \quad \text { q.c. },
$$

ou seja, $\left(\theta_{t} \tau \mid \Im_{t}\right) \downarrow$ st $t$.

A relação entre a definição clássica de IFR e esse novo conceito é dada pela seguinte proposição :

Proposição 2.2.2: Um tempo de vida $\tau$ tem a propriedade IFR se e somente se $\tau$ é IFR $\mid \sigma\left\{1_{\{\tau>s\}} ; s \leq t\right\}$.

Prova: Consideremos a função

$$
f(t, s, \omega)=\left\{\begin{array}{cl}
\frac{\bar{F}(t+s)}{\bar{F}(t)}, & \text { se } \bar{F}(t)>0 \text { e } \omega \in\{\tau>t\}, \\
0, & \text { caso contrário. }
\end{array}\right.
$$

Notemos que um conjunto em $\Im_{t}$ é da forma $C=\{\tau>u\}, u \leq t$ e assim, 


$$
\begin{aligned}
\int_{C} f(t, s, \omega) P(d \omega) & =\int_{\{\tau>u\}} \frac{\bar{F}(t+s)}{\bar{F}(t)} 1_{\{\tau>t\}}(\omega) \mathbb{P}(d \omega) \\
& =\frac{\bar{F}(t+s)}{\bar{F}(t)} \int_{\Omega} 1_{\{\tau>t\}}(\omega) \mathbb{P}(d \omega) \\
& =\bar{F}(t+s) \\
& =P\{\tau>t+s, \tau>u\} \\
& =P\left\{\theta_{t} \tau>s, C\right\}
\end{aligned}
$$

Portanto, $f(t, s, \omega)$ é uma versão de $P\left\{\theta_{t} \tau>s \mid \Im_{t}\right\}$ e os dois conceitos são equivalentes.

A extensão multivariada desse novo conceito é natural.

Definição 2.2.3. Dizemos que $\mathrm{S}=\left(S_{i}\right)_{1 \leq i \leq n}$ tem taxa de falha crescente relativa

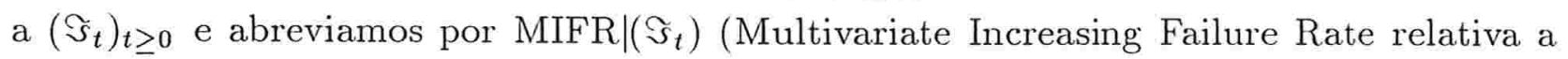
$\left.\left(\Im_{t}\right)_{t \geq 0}\right)$ se, para todo conjunto superior aberto $U \in \mathcal{U}$ e todo $0 \leq t^{*} \leq t$, tivermos

$$
\mathbb{P}\left\{\theta_{t} \mathrm{~S} \in U \mid \Im_{t}\right\} \leq \mathbb{P}\left\{\theta_{t^{*}} \mathrm{~S} \in U \mid \Im_{t^{*}}\right\} \quad \text { q.c. . }
$$

Dizemos que $\mathbf{S}$ tem essa propriedade fracamente (weakly) e escrevemos WMIFR se $U$ é restrito à classe dos conjuntos superiores diagonais. Os conceitos de conjuntos superiores e conjuntos superiores diagonais são encontrados no Apêndice.

Podemos provar que $\mathrm{S}=\left(S_{i}\right)_{1 \leq i \leq n}$ é MIFR $\mid\left(\Im_{t}\right)$ se e somente se existe uma versão regular da distribuição condicional $\mathbb{I P}$ e um conjunto de medida nula $N$, tal que em $N^{c}$

$$
\mathbb{P}\left\{\theta_{t} \mathrm{~S} \in U \mid \Im_{t}\right\} \leq \mathbb{P}\left\{\theta_{t^{*}} \mathrm{~S} \in U \mid \Im_{t^{*}}\right\}
$$

para todo $0 \leq t^{*} \leq t$ e todo conjunto superior $U \in \mathcal{U}$.

Como qualquer esperança condicional é igual (q.c.) a uma integral com respeito a uma versão regular da probabilidade condicional, podemos enunciar a equivalência: 
$\mathrm{S}$ é $\operatorname{MIFR} \mid\left(\Im_{t}\right)$ se e somente se, para toda função $f: \mathbb{R}^{n} \rightarrow \mathbb{R}$ Borel mensurável, crescente e limitada tivermos

$$
\mathbb{E}\left[f\left(\theta_{t} \mathrm{~S}\right) \mid \Im_{t}\right] \leq \mathbb{E}\left[f\left(\theta_{t^{*}} \mathrm{~S}\right) \mid \Im_{t^{*}}\right] \quad \text { q.c. },
$$

para todo $0 \leq t^{*} \leq t$

Discutiremos agora a preservação da propriedade (W)MIFR $\mid\left(\Im_{t}\right)$ sobre a formação de sistemas monótonos. Provaremos que a propriedade MIFR $\mid\left(\Im_{t}\right)$ dos tempos de vida dos componentes é preservada para o tempo de vida do sistema se a família de $\sigma$-álgebra $\left(\Im_{t}\right)_{t \geq 0}$ for mantida. Começaremos, entretanto, com um caso um pouco mais geral.

Suponhamos que os componentes $C_{i}$ com tempos de vida $S_{i}(1 \leq i \leq n)$ são usados para formar $m$ sistemas monótonos binários $\phi_{j}, 1 \leq j \leq m$. Cada componente $C_{i}$ pode fazer parte de mais de um sistema $\phi_{j}$ ao mesmo tempo e alguns componentes podem não fazer parte de sistema algum. Denotando o tempo de vida de $\phi_{j}$ por $\tau_{j}$ temos

$$
\tau_{j}=\min _{k} \max _{i \in K_{j k}} S_{i}
$$

onde $K_{j k}$ denota o $k$-ésimo conjunto de corte de $\phi_{j}$.

Teorema 2.2.4: Nas condições anteriores, se $\mathrm{S}=\left(S_{i}\right)_{1 \leq i \leq n}$ é $\operatorname{MIFR} \mid\left(\Im_{t}\right)$ então $\vec{\tau}=\left(\tau_{j}\right)_{1 \leq j \leq m}$ é $\operatorname{MIFR} \mid\left(\Im_{t}\right)$.

Prova: Se escrevermos $\tau_{j}=\tau_{j}(\mathbf{S})$, então:

$$
\begin{aligned}
\theta_{t} \tau_{j}(\mathrm{~S}) & =\left(\tau_{j}(\mathrm{~S})-t\right)^{+} \\
& =\left(\left(\min _{k} \max _{i \in K_{j k}} S_{i}\right)-t\right)^{+}=\left(\min _{k} \max _{i \in K_{j k}}\left(S_{i}-t\right)\right)^{+} \\
& =\max _{k} \min _{i \in K_{j k}}\left(S_{i}-t\right)^{+}=\tau_{j}\left(\theta_{t} \mathrm{~S}\right) .
\end{aligned}
$$

E mais, a função $\mathrm{S} \rightarrow \vec{\tau}(\mathrm{S})$ é crescente em $\mathrm{S}$. Então, se $f: \mathbb{R}^{m} \rightarrow \mathbb{R}^{1}$ é crescente, temos que

$$
f\left(\theta_{t} \vec{\tau}\right)=f\left(\vec{\tau}\left(\theta_{t} \mathrm{~S}\right)\right)=(f \circ \vec{\tau})\left(\theta_{t} \mathrm{~S}\right),
$$


onde a função composta $f \circ \vec{\tau}: \mathbb{R}^{n} \rightarrow \mathbb{R}^{1}$ é crescente. Assim, como por hipótese S é $\operatorname{MIFR} \mid\left(\Im_{t}\right)$,

$$
\mathbb{E}\left((f \circ \vec{\tau})\left(\theta_{t} \mathrm{~S}\right) \mid \Im_{t}\right) \leq \mathbb{E}\left((f \circ \vec{\tau})\left(\theta_{t^{*}} \mathrm{~S}\right) \mid \Im_{t^{*}}\right) \quad \text { q.c. }
$$

para $0 \leq t^{*} \leq t$, o que implica que

$$
\mathbb{E}\left(f\left(\theta_{t} \vec{\tau}\right) \mid \Im_{t}\right) \leq \mathbb{E}\left(f\left(\theta_{t^{*}} \vec{\tau}\right) \mid \Im_{t^{*}}\right) \quad \text { q.c. }
$$

e provamos que $\vec{\tau}$ é $\operatorname{MIFR} \mid\left(\Im_{t}\right)$.

Corolário 2.2.5: Se $\mathrm{S}=\left(S_{i}\right)_{1 \leq i \leq n}$ é MIFR $\mid\left(\Im_{t}\right)$, então qualquer tempo de vida $\tau=\tau(\mathbf{S})$ de um sistema monótono com componentes $C_{i}$ é $\operatorname{IFR} \mid\left(\Im_{t}\right)$.

O corolário acima parece contradizer uma propriedade bem conhecida: os sistemas monótonos formados por componentes independentes com tempo de vida IFR não tem necessariamente um tempo de vida IFR (Barlow e Proschan (1981)). Consideremos o seguinte exemplo para elucidar a questão:

Exemplo 2.2.6 (Barlow e Proschan (1981), p.83): Sejam $C_{1}$ e $C_{2}$ dois componentes exponenciais independentes de parâmetro 1, em paralelo. Aplicando o corolário temos que $\tau=S_{1} \vee S_{2}$ é IFR $\mid \sigma\left(1_{\left\{S_{i} \geq s\right\}}, s \leq t, i=1,2\right)$, mas não podemos fazer afirmações (usando o corolário) a respeito de $\tau$ em relação a $\sigma\left(1_{\{\tau \geq s\}}, s \leq t\right.$ ); na verdade sabemos que $\tau$ não é IFR $\mid \sigma\left(1_{\{\tau \geq s\}}, s \leq t\right)$, ou seja, IFR.

Um outro resultado interessante é obtido quando fazemos a recíproca do corolário anterior:

Proposição 2.2.7: Suponhamos que $\tau$ seja $\operatorname{IFR} \mid\left(\Im_{t}\right)$ para qualquer sistema monótono formado por $C_{1}, \ldots, C_{n}$. Então $\mathrm{S}$ é WMIFR|( $\left.\Im_{t}\right)$.

Prova: Seja $U \in \mathcal{U}(d)$ um conjunto diagonal arbitrário, com $d \in \mathbb{R}^{1}$. Então, pelo Lema (A.1.5), $U$ pode ser escrito como:

$$
\begin{gathered}
U=\left\{\mathrm{x} \in R^{n}: \max _{1 \leq k \leq k_{0}} \min _{i \in P_{k}} x_{i}>d\right\}, \\
-42-
\end{gathered}
$$


onde $1 \leq k_{0} \leq 2^{n}-1$ e $P_{k} \subset\{1,2, \ldots, n\}\left(1 \leq k \leq k_{0}\right)$. Se $\phi$ for um sistema com tempo de vida $\tau=\max _{1 \leq k \leq k_{0}} \min _{i \in P_{k}} T_{i}$ (basta tomar os conjuntos $P_{k}$ como conjuntos de caminho), temos

$$
\begin{aligned}
\left\{\theta_{t} \mathrm{~S} \in U\right\} & =\left\{\max _{1 \leq k \leq k_{0}} \min _{i \in P_{k}}\left(S_{i}-t\right)^{+}>d\right\} \\
& =\left\{\left(\max _{1 \leq k \leq k_{0}} \min _{i \in P_{k}} S_{i}-t\right)^{+}>d\right\} \\
& =\left\{\theta_{t} \tau>d\right\} .
\end{aligned}
$$

Assim,

$$
\begin{gathered}
\mathbb{P}\left\{\theta_{t} \mathrm{~S} \in U\right\}=\mathbb{P}\left\{\theta_{t} \tau>d\right\} \leq \\
\leq \mathbb{P}\left\{\theta_{t^{*}} \tau>d\right\}=\mathbb{P}\left\{\theta_{t^{*}} \mathrm{~S} \in U\right\} \quad \text { q.c. },
\end{gathered}
$$

para $0 \leq t^{*} \leq t$ e $U$ conjunto superior diagonal.

Já demonstramos que a formação de sistemas monótonos preserva a propriedade $\operatorname{MIFR} \mid\left(\Im_{t}\right)$ e mostramos que o mesmo não acontece se mudamos a família de $\sigma$-álgebras. Seria desejável encontrar condições sob as quais sistemas monótonos preservassem a propriedade $\operatorname{MIFR} \mid\left(\Im_{t}\right)$ de seus componentes, mas relativamente a uma família de $\sigma$-álgebras $\left(\mathcal{G}_{t}\right)_{t \geq 0}$ contida na família $\left(\Im_{t}\right)_{t \geq 0}$. Como exemplo, consideremos

$\Im_{t}=\sigma\left\{1_{\left\{S_{i}>s\right\}}, s \leq t, 1 \leq i \leq n\right\}$ a $\sigma$-álgebra gerada pelos indicadores dos componentes e

$\mathcal{G}_{t}=\sigma\left\{1_{\left\{\tau_{j}>s\right\}}, s \leq t, 1 \leq j \leq m\right.$ ), a $\sigma$-álgebra gerada pelos indicadores dos sistemas.

Notemos que $\tau_{j}>s=\bigcap_{k} \bigcup_{i \in K_{j k}}\left\{S_{i}>s\right\}$ de maneira que $\mathcal{G}_{t} \subset \Im_{t}, t \geq 0$.

Essas condições estão no seguinte teorema:

Teorema 2.2.8: Suponhamos que $\mathrm{S}$ seja (W)MIFR $\mid\left(\Im_{t}\right)$ e que $\mathcal{G}_{t} \subset \Im_{t}$ seja tal que, para todo $t \geq 0, \theta_{t} \vec{\tau}$ seja independente de $\Im_{t}$, dado $\mathcal{G}_{t}$. Então $\vec{\tau}$ é $(\mathrm{W}) \operatorname{MIFR} \mid\left(\mathcal{G}_{t}\right)$.

Prova: Pelo Teorema 2.2.4. $\vec{\tau}$ é $(W) M I F R \mid\left(\Im_{t}\right)$ de maneira que, para todo conjunto superior aberto (diagonal) $U$ e $0 \leq t^{*} \leq t$, 


$$
\mathbb{P}\left(\theta_{t} \vec{\tau} \in U \mid \Im_{t}\right) \leq \mathbb{P}\left(\theta_{t^{*}} \vec{\tau} \in U \mid \Im_{t^{*}}\right) \quad \text { q.c. . }
$$

Como $\vec{\tau}$ é independente de $\Im_{t}$, dado $\mathcal{G}_{t}$, podemos afirmar que

$$
\begin{aligned}
\mathbb{P}\left(\theta_{t} \vec{\tau} \in U \mid \mathcal{G}_{t}\right) & =\mathbb{P}\left(\theta_{t} \vec{\tau} \in U \mid \Im_{t}\right) \\
& \leq \mathbb{P}\left(\theta_{t^{*}} \vec{\tau} \in U \mid \Im_{t^{*}}\right) \\
& =\mathbb{P}\left(\theta_{t^{*}} \vec{\tau} \in U \mid \mathcal{G}_{t^{*}}\right) \quad \text { q.c. }
\end{aligned}
$$

Um exemplo específico da aplicação desse teorema é dado pelo seguinte corolário:

Corolário 2.2.9: Seja $\tau$ o tempo de vida de um sistema em série cujos tempos de vida dos componentes S é MIFR $\mid\left(\Im_{t}\right)$, onde $\Im_{t}=\sigma\left\{1_{\left\{S_{i}>s\right\}}, s \leq t, 1 \leq i \leq n\right\}$. Então $\tau$ é IFR.

Prova: Como IFR é o mesmo que IFR $\mid\left(\mathcal{G}_{t}\right)$, onde $\mathcal{G}_{t}=\sigma\left\{1_{\{r>s\}}, s \leq t\right\}$, devemos mostrar que, para todo $s$ e $t \geq 0,\left\{\theta_{t} \tau>s\right\}$ é independente de $\Im_{t}$, dado $\mathcal{G}_{t}$. Como $\mathcal{G}_{t} \subset \Im_{t}$, é suficiente provar que

$$
\mathbb{P}\left\{\theta_{t} \tau>s \mid \Im_{t}\right\}=\mathbb{P}\left\{\theta_{t} \tau>s \mid \mathcal{G}_{t}\right\} \quad \text { q.c. }
$$

Seja então

$$
f(t, s ; \omega)=\left\{\begin{array}{cl}
\frac{\bar{F}((t+s) 1)}{\bar{F}(t 1)}, & \text { se } \omega \in\left\{S_{i}>t, 1 \leq i \leq n\right\} \text { e } \bar{F}(t 1)>0 \\
0, & \text { caso contrário. }
\end{array}\right.
$$

Se $C \in \Im_{t}, C$ é da forma $\{\mathbf{S}>\mathbf{u}\}$ para algum $\mathbf{u} \leq t \mathbf{1}$

$$
\begin{aligned}
\int_{C} f(t, s ; \omega) P(d \omega) & =\int_{\{\mathrm{S}>\mathrm{u}\}} \frac{\bar{F}((t+s) 1)}{\bar{F}(t 1)} 1_{\{\mathrm{S}>t 1\}}(\omega) P(d \omega) \\
& =\frac{\bar{F}((t+s) 1)}{\bar{F}(t \mathbf{1})} \int_{\Omega} 1_{\{\mathrm{S}>t 1\}}(\omega) P(d \omega) \\
& =\bar{F}((t+s) \mathbf{1}) \\
& =\mathbb{P}\{\mathrm{S}>(t+s) \mathbf{1} ; \mathrm{S}>\mathbf{u}\} \\
& =\mathbb{P}\left\{\theta_{t} \tau>s, C\right\} \\
& \quad-44-
\end{aligned}
$$


e concluímos que $f(t, s ; \omega)$ é uma versão de $\mathbb{P}\left\{\theta_{t} \tau>s \mid \Im_{t}\right\}$. Como os conjuntos em $\mathcal{G}_{t}$ são da forma $\{\mathrm{S}>s 1\}$, para algum $s \leq t, f(t, s, \omega)$ é também uma versão de $\mathbb{P}\left\{\theta_{t} \tau>s \mid \mathcal{G}_{t}\right\}$.

Estes novos conceitos preservam a maioria das propriedades que poderiam ser chamadas de desejáveis para qualquer extensão do conceito clássico de confiabilidade. Já verificamos na Proposição 2.2 .2 como a classe $\operatorname{IFR} \mid\left(\Im_{t}\right)$ se reduz à classe de distribuições IFR, em um caso especial. Mostraremos agora que qualquer subvetor de um vetor $\operatorname{MIFR} \mid\left(\Im_{t}\right)$ também é $\operatorname{MIFR} \mid\left(\Im_{t}\right)$ e que a composição de dois vetores $\operatorname{MIFR} \mid\left(\Im_{t}\right)$, independentes, é $\operatorname{MIFR} \mid\left(\Im_{t}\right)$.

Teorema 2.2.10: Suponhamos que $\mathrm{S}=\left(S_{i}\right)_{1 \leq i \leq n}$ é MIFR $\mid\left(\Im_{t}\right)$. Então, qualquer subvetor $S_{o}=\left(S_{i}\right)_{i \in I_{o}} \operatorname{com} I_{o} \subset\{1,2, \ldots, n\}$ é $\operatorname{MIFR} \mid\left(\Im_{t}\right)$. Em particular, cada $S_{i}$ é $\operatorname{IFR} \mid\left(\Im_{t}\right)$.

Prova: Segue facilmente pois qualquer conjunto superior $U_{0}$ em $\mathbb{R}^{\left.\text {card( } I_{0}\right)}$ pode ser escrito como $U=\left\{\mathrm{x} \in \mathbb{R}^{n}:\left(x_{i}\right)_{i \in I_{0}} \in U_{0}\right\}$, um conjunto superior em $\mathbb{R}^{n}$ e portanto, se $0 \leq t^{*} \leq t$, temos que

$$
\begin{gathered}
\mathbb{P}\left\{\theta_{t} \mathrm{~S}_{0} \in U_{0} \mid \Im_{t}\right\}=\mathbb{P}\left\{\theta_{t} \mathrm{~S} \in U \mid \Im_{t}\right\} \leq \\
\mathbb{P}\left\{\theta_{t^{*}} \mathrm{~S} \in U \mid \Im_{t^{*}}\right\}=\mathbb{P}\left\{\theta_{t^{*}} \mathrm{~S}_{0} \in U_{0} \mid \Im_{t^{*}}\right\} \text { q.c. } .
\end{gathered}
$$

No teorema anterior, a família de sub $\sigma$-álgebras $\left(\Im_{t}\right)_{t \geq 0}$ é a mesma para $\mathbf{S}$ e $\mathbf{S}_{\mathbf{0}}$. Seria interessante verificar se $\left(\Im_{t}\right)_{t \geq 0}$ pode ser alterada para uma família de $\sigma$-álgebras $\left(\mathcal{G}_{t}\right)_{t \geq 0} \operatorname{com} \mathcal{G}_{t} \subset \Im_{t}$, para todo $t \geq 0$, com o propósito de analisarmos $\left(\theta_{t} \mathbf{T}_{\mathbf{0}} \mid \mathcal{G}_{t}\right)$.

Note que, em geral, se $\mathcal{G}_{t} \subset \Im_{t}$, a implicação $\mathrm{S}$ é $\operatorname{MIFR} \mid\left(\Im_{t}\right) \Rightarrow \mathrm{S}$ é $\operatorname{MIFR} \mid\left(\mathcal{G}_{t}\right)$ não é verdadeira; basta lembrarmos que sistemas monótonos de componentes $\operatorname{MIFR} \mid\left(\Im_{t}\right)$ é $\operatorname{MIFR} \mid\left(\Im_{t}\right)$ mas não é $\operatorname{MIFR} \mid\left(\mathcal{G}_{t}\right)$, onde

$$
\Im_{t}=\sigma\left\{1\left\{S_{i}>s\right\}, s \leq t, 1 \leq i \leq n\right\} \text { e } \mathcal{G}_{t}=\sigma\left\{1\left\{\tau_{j}>s\right\}, s \leq t, 1 \leq j \leq m\right\} .
$$

A proposição abaixo descreve um caso em que vale esta afirmação. Basicamente assumimos que, devido a independência, uma parte da $\sigma$-álgebra é redundante para o subvetor. 
Proposição 2.2.11: Suponhamos que $\mathrm{S}=\left(S_{i}\right)_{1 \leq i \leq n}$ seja $\operatorname{MIFR} \mid\left(\Im_{t}\right)$ onde $\Im_{t}$ é a $\sigma$-álgebra gerada pelos indicadores dos componentes. Seja $I_{0} \subset\{1,2, \ldots, n\}$ e $\mathcal{G}_{t}$ a $\sigma$-álgebra gerada pelos indicadores dos componentes que pertencem a $I_{0}$. Se $\mathrm{S}_{\mathbf{0}}=\left(S_{i}\right)_{i \in I_{0}}$ e $\mathrm{S}_{1}=\left(S_{i}\right)_{i \in I_{0}^{c}}$ são independentes, então $\mathrm{S}_{0}$ é $\operatorname{MIFR} \mid\left(\mathcal{G}_{t}\right)$.

Prova: Seja $\mathcal{K}_{t}=\sigma\left\{1_{\left\{S_{i}>s\right\}}, s \leq t, i \in I_{0}^{c}\right\}$, assim $\Im_{t}=\mathcal{G}_{t} \vee \mathcal{K}_{t}$. Denotemos a independência de $\mathcal{A}$ e $\mathcal{B}$ por $\mathcal{A} \coprod \mathcal{B}$ e a independência condicional, dado $\mathcal{C}$, por $\mathcal{A} \amalg \mathcal{B} \mid \mathcal{C}$.

Por hipótese $\mathcal{G}_{\infty} \coprod \mathcal{K}_{\infty}$ e portanto $\mathcal{G}_{\infty} \amalg \mathcal{K}_{t}$. Pelo fato de que $\mathcal{G}_{t} \subset \mathcal{G}_{\infty}$ temos então que $\mathcal{G}_{\infty} \coprod \mathcal{K}_{t} \mid \mathcal{G}_{t}$ (pois para qualquer $B \in \mathcal{K}_{t}, \mathbb{P}\left(B \mid \mathcal{G}_{t} \vee \mathcal{G}_{\infty}\right)=\mathbb{P}\left(B \mid \mathcal{G}_{\infty}\right)=\mathbb{P}(B)=$ $=\mathbb{P}\left(B \mid \mathcal{G}_{t}\right)$ q.c. $)$.Portanto, para qualquer $A \in \mathcal{G}_{\infty}$, temos $\mathbb{P}\left(A \mid \mathcal{G}_{t} \vee \mathcal{K}_{t}\right)=\mathbb{P}\left(A \mid \mathcal{G}_{t}\right)$ q.c. e em particular, temos que

$$
\mathbb{P}\left(\theta_{t} \mathrm{~S}_{0} \in U \mid \Im_{t}\right)=\mathbb{P}\left(\theta_{t} \mathrm{~S}_{\mathbf{0}} \in U \mid \mathcal{G}_{t}\right) \quad \text { q.c. . }
$$

Similarmente,

$$
\mathbb{P}\left(\theta_{t^{*}} \mathrm{~S}_{0} \in U \mid \Im_{t^{*}}\right)=\mathbb{P}\left(\theta_{t^{*}} \mathrm{~S}_{0} \in U \mid \mathcal{G}_{t^{*}}\right) \quad \text { q.c. }
$$

e portanto, pelo Teorema 2.2.10,

$$
\mathbb{I P}\left(\theta_{t} \mathrm{~S}_{\mathbf{0}} \in U \mid \mathcal{G}_{t}\right) \leq \mathbb{P}\left(\theta_{t^{*}} \mathrm{~S}_{\mathbf{0}} \in U \mid \mathcal{G}_{t^{*}}\right) \quad \text { q.c. }
$$

se $0 \leq t^{*} \leq t$, onde $U$ é um conjunto superior aberto.

O próximo teorema mostra que se dois vetores independentes são, separadamente, $\operatorname{MIFR} \mid\left(\Im_{t}\right)$ então a junção dos dois vetores também é $\operatorname{MIFR} \mid\left(\Im_{t}\right)$.

Teorema 2.2.12: Suponhamos que $S_{1}$ e $S_{2}$ sejam MIFR $\mid \Im_{t}$ e que para cada t, $\theta_{t} \mathbf{S}_{1}$ e $\theta_{t} \mathbf{S}_{2}$ sejam independentes, dado $\Im_{t}$. Então $\mathbf{S}=\left(\mathbf{S}_{1}, \mathbf{S}_{2}\right)$ também é MIFR $\mid \Im_{t}$.

Prova: Para $\omega \notin N$ ( $N$ conjunto de medida nula), escrevamos a versão regular da probabilidade condicional

$$
\begin{gathered}
\mathbb{P}^{*}\left(\theta_{u} \mathrm{~S}_{\mathbf{i}} \in \cdot \mid \Im_{u}\right)(\omega)=\mathrm{H}_{\mathbf{i}}(u, \cdot ; \omega), \quad i=1,2 . \\
-46-
\end{gathered}
$$


Seja $d_{i}=\operatorname{card}\left(I_{i}\right), i=1,2$ e seja $f$ uma função crescente $f: \mathbb{R}^{d_{1}+d_{2}} \rightarrow \mathbb{R}^{1}$. Assim, para $0 \leq t^{*} \leq t$,

$$
\begin{aligned}
\mathbb{E}\left(f\left(\theta_{t}\left(\mathbf{S}_{1}, \mathbf{S}_{2}\right)\right) \mid \Im_{t}\right) & =\int\left[\int f\left(\mathbf{x}_{1}, \mathbf{x}_{2}\right) \mathbf{H}_{1}\left(t, d \mathbf{x}_{1} ; .\right)\right] \mathbf{H}_{2}\left(t, d \mathbf{x}_{2} ; .\right) \\
& \leq \int\left[\int f\left(\mathbf{x}_{1}, \mathbf{x}_{2}\right) \mathbf{H}_{1}\left(t^{*}, d \mathbf{x}_{1} ; .\right)\right] \mathbf{H}_{2}\left(t, d \mathbf{x}_{2} ; .\right) \\
& \leq \int\left[\int f\left(\mathbf{x}_{1}, \mathbf{x}_{2}\right) \mathbf{H}_{1}\left(t^{*}, d \mathbf{x}_{1} ; .\right)\right] \mathbf{H}_{2}\left(t^{*}, d \mathbf{x}_{2} ; .\right) \\
& =E\left(f\left(\theta_{t^{*}}\left(\mathbf{S}_{1}, \mathbf{S}_{2} \mid \Im_{t^{*}}\right)\right)\right) .
\end{aligned}
$$

A primeira desigualdade vem do fato de que $f\left(\mathrm{x}_{1}, \mathrm{x}_{2}\right)$ é crescente em $\mathrm{x}_{1}$ para $\mathbf{x}_{2}$ fixado, $\int f\left(\mathbf{x}_{1}, \mathbf{x}_{2}\right) \mathbf{H}_{1}\left(u, d \mathbf{x}_{1} ; \cdot\right)=\mathbb{E}\left(f\left(\theta_{u} \mathrm{~S}_{1}, \mathbf{x}_{2} \mid \Im_{u}\right)\right.$ q.c., e $\mathrm{S}_{1}$ é MIFR $\mid\left(\Im_{t}\right)$. A segunda desigualdade ocorre pois $\int f\left(\mathbf{x}_{1}, \mathbf{x}_{2}\right) \mathbf{H}_{1}\left(t^{*}, d \mathbf{x}_{1} ; \cdot\right)$ é crescente em $\mathbf{x}_{2}$ e $\mathbf{T}_{2}$ é MIFR $\left(\Im_{t}\right)$.

O teorema anterior exige uma única família de $\sigma$-álgebras para $\mathbf{S}=\left(\mathbf{S}_{1}, \mathbf{S}_{2}\right), \mathbf{S}_{1}$ e $S_{2}$. Entretanto, uma situação mais interessante ocorre quando consideramos $\mathbf{S}_{1}$ e $\mathbf{S}_{2}$ em relação as "suas próprias" $\sigma$-álgebras que são combinadas para gerar a $\sigma$-álgebra de $\mathrm{S}=\left(\mathrm{S}_{1}, \mathrm{~S}_{2}\right)$.

Proposição 2.2.13: Suponhamos que $\mathrm{S}_{1}=\left(S_{i}\right)_{i \in I_{1}} \quad$ e $\mathrm{S}_{2}=\left(S_{i}\right)_{i \in I_{2}} \quad$ sejam independentes. Se $\mathrm{S}_{\mathrm{j}}$ é $\operatorname{MIFR} \mid\left(\Im_{t}^{j}\right)$ onde $\Im_{t}^{j} \subset \mathcal{G}_{t}^{j}=\sigma\left\{1_{\left\{S_{i}>s\right\}}, 0 \leq s \leq t, i \in I_{j}\right\}, j=1,2$, então $\mathrm{S}=\left(\mathrm{S}_{1}, \mathrm{~S}_{2}\right)$ é $\operatorname{MIFR} \mid\left(\Im_{t}^{1} \vee \Im_{t}^{2}\right)$.

Prova: Como na proposição anterior, temos que $\mathcal{G}_{\infty}^{1} \amalg \mathcal{G}_{\infty}^{2}$ então $\mathcal{G}_{\infty}^{1} \amalg \Im_{t}^{2}$ e $\mathcal{G}_{\infty}^{1} \coprod \Im_{t}^{2} \mid \Im_{t}^{1}$ e portanto

$$
\mathbb{P}\left(\theta_{t} \mathrm{~S}_{1} \in U \mid \Im_{t}^{1} \vee \Im_{t}^{2}\right)=\mathbb{P}\left(\theta_{t} \mathrm{~S}_{1} \in U \mid \Im_{t}^{1}\right) \text { q.c. }
$$

e, de maneira análoga,

$$
\mathbb{P}\left(\theta_{t} \mathrm{~S}_{2} \in U \mid \Im_{t}^{1} \vee \Im_{t}^{2}\right)=\mathbb{P}\left(\theta_{t} \mathrm{~S}_{2} \in U \mid \Im_{t}^{2}\right) \text { q.c. }
$$

Portanto $\mathrm{S}_{1}$ e $\mathrm{S}_{2}$ são MIFR|( $\left.\Im_{t}^{1} \vee \Im_{t}^{2}\right)$ e $\operatorname{como} \mathcal{G}_{\infty}^{1} \amalg \mathcal{G}_{\infty}^{2}$ implica $\mathcal{G}_{\infty}^{1} \amalg \mathcal{G}_{\infty}^{2} \mid \Im_{t}^{1} \vee \Im_{t}^{2}$, podemos aplicar o teorema anterior e obter o resultado desejado. 
Corolário 2.2.14: Suponhamos que $S_{1}, \ldots, S_{n}$ sejam independentes e IFR. Então $\mathrm{S}=\left(S_{i}\right)_{1 \leq i \leq n}$ é $\operatorname{MIFR} \mid\left(\sigma\left\{1_{\left\{S_{i}>s\right\}}, 0 \leq s \leq t, 1 \leq i \leq n\right)\right)$, ou seja, um conjunto de tempos IFR independentes é MIFR com relação a $\sigma$-álgebra gerada pelos componentes. 


\subsection{Classe de Distribuições MNBU $\mid\left(\Im_{t}\right)$.}

O tempo de vida de um objeto $\tau$ é NBU (New Better than Used) se a variável aleatória $(\tau-t \mid\{\tau>t\})$, condicionada em $\{\tau>t\}$, é estocasticamente menor do que $\tau$. A extensão dessa definição segue das mesmas idéias utilizadas na definição de $\operatorname{MIFR} \mid\left(\Im_{t}\right)$.

Definição 2.3.1: Dizemos que $\mathrm{S}=\left(S_{i}\right)_{1 \leq i \leq n}$ é "Multivariate New Better than Used" relativa à família $\left(\Im_{t}\right)$ e abreviamos por $\mathrm{MNBU}\left(\Im_{t}\right)$ se, para todo conjunto superior aberto $U \in \mathcal{U}$ e para todo $t \geq 0$

$$
\mathbb{P}\left(\theta_{t} \mathrm{~S} \in U \mid \Im_{t}\right) \leq \mathbb{P}\left(\mathrm{S} \in U \mid \Im_{0}\right) \quad \text { q.c. . }
$$

Dizemos que $\mathbf{S}$ tem essa propriedade fracamente (WMNBU) se $U$ é restrito à classe dos conjuntos superiores diagonais.

No caso univariado, dizemos que $\tau$ é $\operatorname{NBU} \mid\left(\Im_{t}\right)_{t \geq 0}$ se, para todo $t \geq 0$ e $s \in \mathbb{R}$

$$
\mathbb{P}\left\{\theta_{t} \tau>s \mid \Im_{t}\right\} \leq \mathbb{P}\left\{\tau>s \mid \Im_{0}\right\} \quad \text { q.c. . }
$$

Em particular, se $\Im_{t}=\sigma\left\{1_{\{\tau>s\}}, s \leq t\right\}$, a definição $\operatorname{NBU} \mid\left(\Im_{t}\right)$ é equivalente à definição clássica NBU. Este fato é facilmente comprovado desde que, como está provado na Proposição 2.2.2,

$$
f(t, s ; \omega)= \begin{cases}\frac{\bar{F}(t+s)}{\bar{F}(t)}, & \text { se } \bar{F}(t)>0 \text { e } \omega \in\{\tau>t\}, \\ 0, & \text { caso contrário, }\end{cases}
$$

é uma versão de $\mathbb{P}\left\{\theta_{t} T>s \mid \Im_{t}\right\}$.

É fácil provar que a classe de distribuições MIFR|( $\left.\Im_{t}\right)\left(\right.$ WMIFR $\left.\mid\left(\Im_{t}\right)\right)$ está contida na classe MNBU|( $\left.\Im_{t}\right)$ (WMNBU|( $\left.\Im_{t}\right)$; basta tomar $t^{*}=0$ na Definição 2.2.1.

Notamos também que todos os resultados provados para a classe de distribuições $\operatorname{MIFR} \mid\left(\Im_{t}\right)$ valem para a classe MNBU| $\left(\Im_{t}\right)$. Contudo, a classe de distribuições MNBU|( $\left.\Im_{t}\right)$ 
é preservada quando restringimos a definição a uma família de $\sigma$-álgebras $\left(\mathcal{G}_{t}\right)_{t \geq 0}$ contida em $\left(\Im_{t}\right)_{t \geq 0}$. (Isto não acontece na classe $\operatorname{MIFR} \mid\left(\Im_{t}\right)$ ).

Teorema 2.3.2: Suponhamos que $\mathrm{S}$ é $\operatorname{MNBU} \mid\left(\Im_{t}\right)$. Se $\left(\mathcal{G}_{t}\right)_{t \geq 0}$ é uma família crescente de $\sigma$-álgebras com $\mathcal{G}_{t} \subset \Im_{t}$, para todo $t>0$ e $\mathcal{G}_{0}=\Im_{0}$, então $\mathrm{S}$ é $\mathrm{MNBU}\left(\mathcal{G}_{t}\right)$.

\section{Prova:}

$$
\begin{aligned}
\mathbb{P}\left\{\theta_{t} \mathrm{~S} \in U \mid \mathcal{G}_{t}\right\} & =\mathbb{E}\left\{\mathbb{P}\left\{\theta_{t} \mathrm{~S} \in U\left|\Im_{t}\right| \mathcal{G}_{t}\right\}\right. \\
& \leq \mathbb{E}\left\{\mathbb{P}\left\{\mathrm{S} \in U\left|\Im_{0}\right| \mathcal{G}_{t}\right\}\right. \\
& =\mathbb{E}\left\{\mathbb{P}\left\{\mathrm{S} \in U\left|\mathcal{G}_{0}\right| \mathcal{G}_{t}\right\}\right. \\
& =\mathbb{P}\left\{\mathrm{S} \in U \mid \mathcal{G}_{0}\right\} .
\end{aligned}
$$

Uma outra propriedade adicional à classe $\mathrm{MNBU} \mid\left(\Im_{t}\right)$ é dada pelo teorema seguinte:

Teorema 2.3.3: Suponhamos que $\mathbf{S}=\left(S_{i}\right)_{1 \leq i \leq n}$ seja $(\mathrm{W}) \mathrm{MNBU} \mid\left(\Im_{t}\right)$, onde $\left(\Im_{t}\right)_{t \geq 0}$ é a família de $\sigma$-álgebras gerada pelos indicadores dos componentes. Se $I_{0}$ é um subconjunto de $\{1,2, \ldots, n\}, \mathbf{S}_{0}=\left(S_{i}\right)_{i \in I_{0}}$ e $\mathcal{G}_{t}$ é a família de $\sigma$-álgebras geradas pelos indicadores dos componentes $\left(S_{i}\right)_{i \in I_{0}}$, então $\mathrm{S}_{\mathbf{0}}$ é $(\mathrm{W}) \mathrm{MNBU}\left(\mathcal{G}_{t}\right)$. Em particular, cada $S_{i}$ é NBU.

Prova: Primeiramente observemos que um conjunto superior $U_{0}$ em $\mathbb{R}^{\operatorname{card}\left(I_{0}\right)}$ pode ser escrito como $U=\left\{\mathrm{x} \in \mathbb{R}^{n}:\left(x_{i}\right)_{i \in I_{0}} \in U_{0}\right\}$ e portanto, para todo $t \geq 0$,

$$
\begin{aligned}
\mathbb{P}\left\{\theta_{t} \mathrm{~S}_{0} \in U_{0} \mid \Im_{t}\right\} & =\mathbb{P}\left\{\theta_{t} \mathrm{~S} \in U \mid \Im_{t}\right\} \\
& \left.\leq \mathbb{P}\left\{\mathrm{S} \in U \mid \Im_{0}\right)\right\} \\
& =\mathbb{P}\left\{\mathrm{S}_{0} \in U_{0} \mid \Im_{0}\right\}
\end{aligned}
$$

e então $\mathrm{S}_{0}$ é $\mathrm{MNBU} \mid\left(\Im_{t}\right)$. Assim, utilizando o Teorema 2.3.2, concluímos que $\mathrm{S}_{0}$ é $\operatorname{MNBU} \mid\left(\mathcal{G}_{t}\right)_{t \geq 0}$.

Notemos que o resultado acima só é válido na classe de distribuições MIFR|( $\left.\Im_{t}\right)$ na presença da independência entre os vetores $\mathbf{S}_{0}=\left(S_{i}\right)_{i \in I_{0}}$ e $\mathbf{S}_{0}^{c}=\left(S_{i}\right)_{i \in I_{0}^{c}}$. 
No caso clássico, a classe das distribuições NBU é fechada por formação de sistemas monótonos. Esta propriedade é preservada na classe MNBU|( $\left.\Im_{t}\right)$, como mostra o teorema seguinte.

Teorema 2.3.4: Suponhamos que S seja (W)MNBU|( $\left.\Im_{t}\right)$ onde $\Im_{t}$ é a $\sigma$-álgebra gerada pelos indicadores dos componentes $S_{i}, 1 \leq i \leq n$. Se $\vec{\tau}=\left(\tau_{j}\right)_{1 \leq j \leq m}$ é um vetor de tempos de vida de sistemas monótonos e $\mathcal{G}_{t}=\sigma\left(1_{\left\{\tau_{j} \geq s\right\}}, 0 \leq s \leq t, 1 \leq j \leq m\right)$, então $\vec{\tau}$ é $(\mathrm{W}) \mathrm{MNBU} \mid\left(\mathcal{G}_{t}\right)$. Em particular, cada $\tau_{j}$ é NBU.

Prova: Como na prova do Teorema 2.2.4, para $f: \mathbb{R}^{m} \rightarrow \mathbb{R}^{1}$ crescente, temos que

$$
f\left(\theta_{t} \vec{\tau}\right)=f\left(\vec{\tau}\left(\theta_{t} \mathbf{S}\right)\right)=(f \circ \vec{\tau})\left(\theta_{t} \mathbf{S}\right)
$$

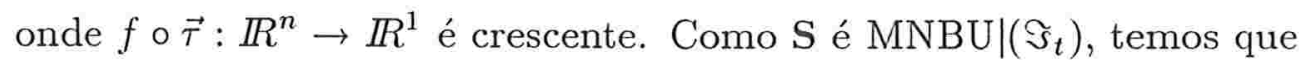

$$
\mathbb{E}\left\{(f \circ \vec{\tau})\left(\theta_{t} \mathbf{S}\right) \mid \Im_{t}\right\} \leq \mathbb{E}\left\{(f \circ \vec{\tau})(\mathbf{S}) \mid \Im_{0}\right\}
$$

e portanto

$$
\mathbb{E}\left\{f\left(\theta_{t} \vec{\tau}\right) \mid \Im_{t}\right\} \leq \mathbb{E}\left\{f(\vec{\tau}) \mid \Im_{0}\right\}
$$

isto é, $\vec{\tau}$ é $\mathrm{MNBU}\left(\Im_{t}\right)$. Como $\mathcal{G}_{t} \subset \Im_{t}$ para todo $t>0$ e $\mathcal{G}_{0}=\Im_{0}$, utilizando o Teorema 2.3.2, concluímos que $\vec{\tau}$ é $\operatorname{MNBU} \mid\left(\mathcal{G}_{t}\right)$. 


\subsection{O Processo de Risco.}

Observando as falhas dos componentes como um processo pontual multivariado $\left(N_{t}(I) ; I \in \Lambda\right)_{t \geq 0}$ analisaremos a noção de risco através dos compensadores desse processo. Começaremos com um conjunto de componentes fixado $I \in \Lambda$, o processo de contagem $\left(N_{t}(I)\right)_{t \geq 0}$ associado a $I$ e introduziremos o correspondente processo de risco. A família de tais processos, indexada por $I \in \Lambda$, é chamada processo de risco multivariado.

Note que $\left(N_{t}(I)\right)_{t \geq 0}$ admite a decomposição de Doob-Meyer

$$
N_{t}(I)=M_{t}(I)+A_{t}(I)
$$

onde $\left(M_{t}(I)\right)_{t \geq 0}$ é um $\Im_{t}$-martingal uniformemente integrável e $\left(A_{t}(I)\right)_{t \geq 0}$ é um processo previsível crescente.

Teorema 2.4.1: Nas condições acima, $A_{t}(I)$ pode ser expressa como

$$
A_{I}(d t)=\sum_{k \geq 0} \frac{G_{k}\left(d t-T_{k}, I\right)}{H_{k}\left(\left[t-T_{k}, \infty\right]\right)} 1_{\left\{T_{k} \leq t \leq T_{k+1}\right\}} \quad \text { q.c. }
$$

onde $G_{k}(d s, I)$ é uma versão regular da distribuição condicional de $\left(T_{k+1}, X_{k+1}\right)$, dado $\Im_{T_{k}}$ e, $H_{k}(d t)=G_{k}(d t, \Lambda)$ é a distribuição condicional de $T_{k+1}-T_{k}$ dado $\Im_{T_{k}}$.

Para provar o teorema precisaremos provar dois lemas:

Lema 2.4.2: Se $T$ é um tempo de parada, para cada $k \leq q$ existirá $R_{k} \in \Im_{T_{k}}$ tal que $T \wedge T_{k+1}=\left(T_{k}+R_{k}\right) \wedge T_{k+1}$ no conjunto $\left\{T_{k} \leq T\right\}$.

Prova: Para cada $s>0$ considere $F_{s}=\left\{T_{k}+s<T_{k+1}\right\}$. A $\sigma$-álgebra $\Im_{\left(T_{k}+s\right)^{-}}$ é gerada pelos conjuntos da forma $D=\left\{N_{r}(I)=p\right\} \cap\left\{r<T_{k}+s\right\}$. Seja

$$
D^{*}=\left\{N_{I}(r)=p\right\} \cap\left\{r<T_{k}\right\}+\left\{N_{I}\left(T_{k}\right)=p\right\} \cap\left\{T_{k} \leq r<T_{k}+s\right\}
$$

então 


$$
\begin{aligned}
D \cap F_{s}= & \left\{N_{I}(r)=p\right\} \cap\left\{r<T_{k}+s\right\} \cap F_{s} \\
= & \left\{N_{I}(r)=p\right\} \cap\left\{r<T_{k}+s\right\} \cap F_{s} \cap\left\{r<T_{k}\right\} \\
& +\left\{N_{I}(r)=p\right\} \cap\left\{r<T_{k}+s\right\} \cap F_{s} \cap\left\{r \geq T_{k}\right\} \\
= & \left\{N_{I}(r)=p\right\} \cap\left\{r<T_{k}\right\} \cap F_{s}+\left\{N_{I}\left(T_{k}\right)=p\right\} \cap \\
& \cap\left\{T_{k} \leq r<T_{k}+s\right\} \cap F_{s} \\
= & \left\{\left\{N_{I}(r)=p\right\} \cap\left\{r<T_{k}\right\}+\left\{N_{I}\left(T_{k}\right)=p\right\} \cap\right. \\
& \left.\cap\left\{T_{k} \leq r<T_{k}+s\right\}\right\} \cap F_{s} \\
= & D^{*} \cap F_{s} .
\end{aligned}
$$

Temos que $D^{*} \in \Im_{T_{k}}$ e $\Im_{\left(T_{k}+s\right)^{-}} \cap F_{s}=\Im_{T_{k}} \cap F_{s}$. Quando $s \downarrow t, \Im_{\left(T_{k}+s\right)^{-}} \downarrow \Im_{T_{k}+t}$ e $F_{s} \uparrow F_{t}$, o que implica que $\Im_{T_{k}+t} \cap F_{t}=\Im_{T_{k}} \cap F_{t}$.

Assim, existe $G_{t} \in \Im_{T_{k}}$ tal que $\left\{T<T_{k}+t\right\} \cap F_{t}=G_{t} \cap F_{t}$ e basta verificar que $R_{k}$ definida como $\left\{R_{k}<t\right\}=\bigcup_{r<t, r \in Q} G_{r}$ satisfaz o lema. Para tanto, provaremos que no conjunto $\left\{T_{k} \leq T\right\}$ vale $\left\{T \wedge T_{k+1}<s\right\}=\left\{T_{k}+R_{k} \wedge T_{k+1}<s\right\}$, para $s \in \mathbb{R}_{+}$.

Se $\omega$ é tal que $T_{k+1}(\omega)<s$, a igualdade é trivial. Se $T_{k+1}(\omega) \geq s$, temos as seguintes equivalências: 


$$
\begin{aligned}
\left\{T_{k}+R_{k} \wedge T_{k+1}<s\right\} & \Leftrightarrow\left\{T_{k}+R_{k}<s\right\} \Leftrightarrow \bigcup_{t<s, t \in Q}\left\{R_{k}<t\right\} \cap\left\{T_{k}<s-t\right\} \\
& \Leftrightarrow \bigcup_{t<s, t \in Q} \bigcup_{r<t, r \in Q} G_{r} \cap\left\{T_{k}<s-t\right\} \\
& \Leftrightarrow \bigcup_{t<s, t \in Q} G_{t} \cap\left\{T_{k}<s-t\right\} \\
& \Leftrightarrow\left(\bigcup_{t<s, t \in Q} G_{t} \cap F_{t} \cap\left\{T_{k}<s-t\right\}\right)+ \\
& \left.+\bigcup_{t<s, t \in Q} G_{t} \cap F_{t}^{c} \cap\left\{T_{k}<s-t\right\}\right) \\
& \Leftrightarrow \bigcup_{t<s, t \in Q} G_{t} \cap F_{t} \cap\left\{T_{k}<s-t\right\} \\
& \Leftrightarrow \bigcup_{t<s, t \in Q}\left\{T<T_{k}+t\right\} \cap\left\{T_{k}<s-t\right\} \\
& \Leftrightarrow\left\{T-T_{k}+T_{k}<s\right\} \\
& \Leftrightarrow\{T<s\} \\
& \Leftrightarrow\left\{T \wedge T_{k}<s\right\} .
\end{aligned}
$$

Lema 2.4.3: Um processo $\left(Z_{t}\right)_{t \geq 0}$ é previsível se e somente se $Z_{0}$ é $\Im_{0}$-mensurável e se, para cada $k$, existe um processo $\left(Y_{t}^{k}\right) \Im_{T_{k}}$-mensurável que satisfaz $Y_{t}^{k}=Z_{T_{k}+t}$ no conjunto $\left\{0 \leq t \leq T_{k+1}-T_{k}\right\}(t \geq 0$ se $k>q)$.

Prova: i) Condição necessária:

Como a $\sigma$-álgebra dos processos previsíveis é gerada pelos conjuntos $B \times\{0\}$, com $B \in \Im_{0}$ e pelos intervalos estocásticos $[0, T]$ onde $T$ é um tempo de parada, é suficiente considerar $Z_{t}$ na forma $Z_{t}=1_{\{t \leq T\}}$.

Pelo Lema 2.4.2, para cada $k$, existe $R_{k} \in \Im_{T_{k}}$ tal que $T_{k+1} \wedge T=\left(T_{k}+R_{k}\right) \wedge T_{k+1}$ no conjunto $\left\{T_{k} \leq T\right\}$. Consideremos o processo $Y_{t}^{k}=1_{\left\{T_{k} \leq T\right\} \cap\left\{t \leq R_{k}\right\}}$. Devemos provar que

$$
\begin{aligned}
Z_{T_{k}+t}=1_{\left\{T_{k}+t \leq T\right\}} & =1_{\left\{T_{k} \leq T\right\} \cap\left\{t \leq R_{k}\right\}}=Y_{t}^{k} \\
& -54-
\end{aligned}
$$


no conjunto $\left\{0 \leq t \leq T_{k+1}-T_{k}\right\}$,

$$
\begin{aligned}
\left\{T_{k} \leq T\right\} \cap\left\{t \leq R_{k}\right\} & \Leftrightarrow\left\{T_{k} \leq T\right\} \cap\left\{T_{k}+t \leq R_{k}+T_{k}\right\} \\
& \Leftrightarrow\left\{T_{k} \leq T\right\} \cap\left\{T_{k}+t \wedge T_{k+1} \leq R_{k}+T_{k} \wedge T_{k+1}\right\} \\
& \Leftrightarrow\left\{T_{k} \leq T\right\} \cap\left\{T_{k}+t \wedge T_{k+1} \leq T \wedge T_{k+1}\right\} \\
& \Leftrightarrow\left\{T_{k}+t \leq T\right\} .
\end{aligned}
$$

Como $\left\{T_{k} \leq T\right\}$ e $R_{k}$ são $\Im_{T_{k}}$-mensuráveis a condição está satisfeita.

ii) Condição suficiente:

Note que os conjuntos $B x\{0\}\left(B \in \Im_{0}\right)$ e $\left.\left.B x\right] T_{k}, T_{k+1}\right]\left(B \in \Im_{T_{k}}, 1 \leq k \leq q\right)$ são previsível. Considerando as hipóteses do lema, podemos escrever

$$
\begin{aligned}
\left\{(\omega, t): Z_{t}(\omega)>a\right\}= & \left\{(\omega, 0): Z_{0}(\omega)>a\right\}+ \\
& +\sum_{k=0}^{q-1}\left\{(\omega, t): T_{k}(\omega)<t \leq T_{k+1}(\omega), Y_{t-T_{k}(\omega)}^{k}(\omega)>a\right\}
\end{aligned}
$$

e concluímos então que $Z_{t}$ é previsível.

Prova do Teorema 2.4.1:

$$
\begin{aligned}
A_{t}(I)= & \int_{0}^{t} \sum_{k \geq 0} \frac{G_{k}\left(d s-T_{k}, I\right)}{H_{k}\left(\left[s-T_{k}, \infty\right]\right)} 1_{\left\{T_{k}<t \leq T_{k+1}\right\}} \\
= & \sum_{k \geq 0} 1_{\left\{T_{k}<t \leq T_{k+1}\right\}}\left(\sum_{p=0}^{k-1} \int_{0}^{T_{p+1}-T_{p}} \frac{G_{p}(d s, I)}{H_{p}([s, \infty])}+\right. \\
& \left.+\int_{0}^{t-T_{k}} \frac{G_{k}(d s, I)}{H_{k}([s, \infty])}\right) .
\end{aligned}
$$

$\operatorname{Assim} A_{0}(I)=0$ e, para $k \leq q$, temos $Y_{t}^{k}=A_{t+T_{k}}(I)=A_{T_{k}}(I)+\int_{0}^{t} \frac{G_{k}(d s, I)}{H_{k}([s, \infty])}$ e então $A_{t}(I)$ é previsível e crescente. 
Resta provar que $\mathbb{E}\left(A_{T}(I)\right)=\mathbb{E}\left(\left(N_{T}(I)\right)\right.$ para qualquer $\Im_{t}$-tempo de parada $T$.

$$
\begin{aligned}
\mathbb{E}\left(1_{\left\{T_{k} \leq T\right\}}\left(A_{T_{k+1} \wedge T}(I)-A_{T_{k}}(I)\right)\right)= & \mathbb{E}\left(1_{\left\{T_{k} \leq T\right\}} \int_{0}^{\left(T_{k+1} \wedge T\right)-T_{k}} \frac{G_{k}(d u, I)}{H_{k}([u, \infty]}\right) \\
= & \mathbb{E}\left(1_{\left\{T_{k} \leq T\right\}} \int_{0}^{\left(T_{k+1} \wedge T_{k}\right)+R_{k}-T_{k}} \frac{G_{k}(d u, I)}{H_{k}([u, \infty]}\right) \\
= & \mathbb{E}\left(1 _ { \{ T _ { k } \leq T \} } \int _ { 0 } ^ { \infty } \left(\frac{G_{k}(d u, I)}{H_{k}([u, \infty]} 1_{\left\{R_{k}<s\right\}} 1_{\left\{u \leq R_{k}\right\}}+\right.\right. \\
& \left.+\int \frac{G_{k}(d u, I)}{H_{k}([u, \infty])} 1_{\left\{R_{k} \geq s\right\}} 1_{\{u \leq s\} \cap\{u<\infty\})}\right) H_{k}(d s) .
\end{aligned}
$$

Note que $\left\{s \leq R_{k}\right\} \cap\{u \leq s\} \equiv\left\{u \leq R_{k}\right\} \cap\{s \geq u\}$ e, pelo Teorema de Fubini, temos

$$
\begin{aligned}
\mathbb{E}\left(1_{\left\{T_{k} \leq T\right\}}\left(A_{T_{k+1} \wedge T}(I)-A_{T_{k}}(I)\right)\right)= & \left.\mathbb{E}\left(1_{\left\{T_{k} \leq T\right\}} \int_{0}^{\infty} \frac{G_{k}(d u, I)}{H_{k}([u, \infty])} 1\left\{u \leq R_{k}\right\} H_{k}(] R_{k}, \infty\right]\right) \\
& \left.\left.+\int_{0}^{\infty} \frac{G_{k}(d u, I)}{H_{k}([u, \infty])} 1_{\{u<\infty\} \cap\left\{u \leq R_{k}\right\}}\right) H_{k}\left(\left[u, R_{k}\right]\right)\right) \\
= & \mathbb{E}\left(1_{\left\{T_{k} \leq T\right\}} \int_{0}^{\infty} G_{k}(d u, I) 1_{\{u<\infty\} \cap\left\{u \leq R_{k}\right\}}\right) \\
= & \mathbb{E}\left(1_{\left\{T_{k} \leq T\right\}}\left(N_{T \wedge T k+1}(I)-N_{T_{k}}(I)\right) .\right.
\end{aligned}
$$

Assim,

$$
\begin{aligned}
\mathbb{E}\left(A_{T}(I)\right) & =\sum_{k \geq 0} \mathbb{E}\left(1_{\left\{T_{k} \leq T\right\}}\left(A_{T_{k+1} \wedge T}(I)-A_{T_{k}}(I)\right)\right) \\
& =\sum_{k \geq 0} \mathbb{E}\left(1_{\left\{T_{k} \leq T\right\}}\left(N_{T_{k+1} \wedge T}(I)-N_{T_{k}}(I)\right)\right) \\
& =\mathbb{E}\left(N_{T}(I)\right) .
\end{aligned}
$$

Observemos que o $k$-ésimo termo de $d A_{t}(I)$ assume um valor não nulo somente no intervalo aleatório $\left(T_{k}, T_{k+1}\right]$ e, neste intervalo, ele expressa a probabilidade condicional de $\left\{T_{k+1} \in d t, X_{k+1}=I\right\}$, dados os tempos de falha precedentes $T_{1}, \ldots, T_{k}$, seus respectivos 
conjuntos de falha $X_{1}, \ldots, X_{k}$ e o fato de que $T_{k+1} \geq t$. Neste sentido, $\left(A_{t}(I): t \geq 0, I \in \Lambda\right)$ expressa somente os riscos que são imediatos ou associados com a próxima falha.

Na Teoria da Confiabilidade, além de analisarmos o processo de falhas dos componentes, estamos interessados na consequência dessas falhas no tempo de vida do sistema. Podemos descrever o tempo de falha do sistema, que depende das falhas acumuladas dos componentes, da seguinte maneira. Seja

$$
D(t)= \begin{cases}X_{1} \cup \ldots \cup X_{k}, & \text { se } T_{k} \leq t<T_{k+1} \\ \emptyset & \text { se } t<T_{1} .\end{cases}
$$

Note que para cada $\omega$ fixado e para cada $t, T_{k}(\omega) \leq t<T_{k+1}(\omega)$, podemos considerar $0<\delta=\frac{T_{k+1}(\omega)-t}{2}$ de maneira que, para qualquer $\epsilon>0, \exists \delta>0$ tal que se $0<s<t<\delta$ temos $d(D(\omega, t)-D(\omega, s))<\epsilon$, assim $\lim _{s \downarrow t} D(\omega, s)=D(\omega, t)$, isto é, $D$ é contínua à direita.

É fácil notar que $D(\omega, t)$ é crescente na ordem parcial de $\Lambda$. Podemos sempre definir $D\left(t^{-}\right)=\lim _{s \uparrow t} D(s)=\bigcup_{s<t} D(s)$.

Seja $\Lambda_{\phi}=\left\{K_{1}, \ldots, K_{k_{0}}\right\}$ o conjunto de todos os conjuntos de corte de $\phi$. Claramente temos que o tempo de vida do sistema é dado por

$$
\begin{aligned}
\tau & =\inf \left\{t \geq 0: D(t) \in \Lambda_{\phi}\right\} \\
& =\min \left\{T_{k}: X_{1} \cup \ldots \cup X_{k} \in \Lambda_{\phi}\right\}
\end{aligned}
$$

O processo pontual multivariado das falhas do sistema é definido por

$$
N_{t}(\phi)=1_{\{t \geq \tau\}}, t \geq 0,
$$

que foi derivado do processo pontual multivariado $\left(T_{k}, X_{k}\right)_{k \geq 1}$.

Em várias situações que ocorrem na prática estamos interessados em determinar, em um instante $t$ fixado, o conjunto de componentes que podem causar a falha imediata do sistema. A classe desses conjuntos 


$$
\Gamma_{\phi}(t)=\left\{I \in \Lambda: D\left(t^{-}\right) \notin \Lambda_{\phi}, D\left(t^{-}\right) \cup I \in \Lambda_{\phi}\right\}
$$

denominaremos classe dos conjuntos críticos para a falha do sistema no instante $t$.

Para cada $\omega$, a função $t \rightarrow \Gamma_{\phi}(t)$ é crescente na ordem parcial de $\Lambda$ para $t \leq \tau$ e contínua à esquerda. Observemos que o sistema falha em $t$ quando algum conjunto crítico em $\Gamma_{\phi}(t)$ ocorre e reciprocamente, isto é,

$$
\{\tau \in d t\}=\bigcup_{I \in \Gamma_{\phi}(t)}\left\{\tau_{I} \in d t\right\}
$$

onde os eventos $\left\{\tau_{I} \in d t\right\}$ são disjuntos. Estudaremos agora o $\left(\Im_{t}\right)$-compensador do processo de contagem das falhas do sistema $\left(N_{t}(\phi)\right)_{t \geq 0}$ denotado por $\left(A_{t}(\phi)\right)_{t \geq 0}$. Obviamente chamaremos este compensador de processo de risco do sistema. O nosso objetivo é obter o processo de risco do sistema $A_{t}(\phi)$ através do processo de risco multivariado dos componentes $\left(A_{t}(I) ; t \geq 0, I \in \Lambda\right)$.

Teorema 2.4.4: Para todo $t \geq 0$,

$$
A_{t}(\phi)=\int_{(0, t]} \sum_{I \in \Gamma_{\phi}(s)} d A_{s}(I) \quad \text { q.c. . }
$$

Prova: É suficiente mostrar que

$$
\mathbb{E}\left(\int_{\mathbb{R}} g(t) d N_{t}(\phi)\right)=\mathbb{E}\left(\int_{\mathbb{R}} g(t) \sum_{I \in \Gamma_{\phi}(t)} d A_{t}(I)\right)
$$

vale para todo processo previsível $g: \Omega \times \mathbb{R} \rightarrow \mathbb{R}$.

Como $A_{t}(I)$ é o $\left(\Im_{t}\right)$-compensador para $N_{t}(I)$ podemos escrever, para todo processo previsível $f: \Omega \times \mathbb{R x} \Lambda \rightarrow \mathbb{R}$,

$$
\mathbb{E}\left(\int_{\mathbb{R}} \sum_{I \in \Lambda} f(t, I) d N_{t}(I)=\mathbb{E}\left(\int_{\mathbb{R}} \sum_{I \in \Lambda} f(t, I) d A_{t}(I)\right) .\right.
$$

Em particular, $f(t, I)=g(t) 1_{\left\{I \in \Gamma_{\phi}(t)\right\}}$ é previsível pois $g$ é previsível e a função indicadora é contínua à esquerda em $t$. Assim, temos que 


$$
\begin{aligned}
\mathbb{E}\left(\int_{\mathbb{R}} g(t) d N_{t}(\phi)\right) & =\mathbb{E}\left(\int_{\mathbb{R}} \sum_{I \in \Lambda} g(t) 1_{\left\{I \in \Gamma_{\phi}(t)\right\}} d N_{t}(I)\right) \\
& =\mathbb{E}\left(\int_{\mathbb{R}} \sum_{I \in \Lambda} g(t) 1_{\left\{I \in \Gamma_{\phi}(t)\right\}} d A_{t}(I)\right) \\
& =\mathbb{E}\left(\int_{\mathbb{R}} g(t) \sum_{\left.I \in \in \Gamma_{\phi}(t)\right\}} d A_{t}(I)\right) .
\end{aligned}
$$

Uma forma explícita para o compensador $A_{\phi}(t)$ pode ser obtida. Vamos definir o tempo de parada $\sigma_{\phi}(I)$ por

$$
\sigma_{\phi}(I)=\inf \left\{t \geq 0: I \in \Gamma_{\phi}(t)\right\} .
$$

Notemos que $\sigma_{\phi}(I)$ é o instante a partir do qual o conjunto de falhas $I$ se torna crítico para o sistema $\phi$. Claramente, se $\sigma_{\phi}(I)$ é finito, ele é algum tempo de falha $T_{k}$.

Observando que $\left\{I \in \Gamma_{\phi}(t)\right\}=\left\{\sigma_{\phi}(I)<t \leq \tau\right\}$ e utilizando o Teorema 2.4.4 concluímos que

$$
A_{t}(\phi)=\sum_{I \in \Lambda}\left[A_{t \wedge \tau}(I)-A_{\sigma_{\phi}(I)}(I)\right]^{+} \text {q.c. . }
$$

Em outras palavras, o risco do sistema é encontrado acumulando-se os riscos dos conjuntos de falhas $I$ entre os tempos $\sigma_{\phi}(I)$ (quando ele se torna crítico) e $\tau$ (quando o sistema falha).

No caso clássico univariado sabemos que se $\tau$ é o tempo de vida de um objeto com função de distribuição absolutamente contínua, a função de risco $R(t)$ caracteriza algumas classes de distribuições, por exemplo:

- $F$ é IFR se e somente se $R(t)$ é convexa;

- $F$ é NBU se e somente se $R(t)$ é superaditiva.

No que segue, analisaremos as relações entre a realização do Processo de Risco e a classe de distribuição $\operatorname{IFR} \mid\left(\Im_{t}\right)\left(\mathrm{NBU}_{\Im_{t}}\right)$ ), do tempo de vida de um sistema. O único resultado alcançado (Arjas, (1981b)) é dado no teorema abaixo: 
Teorema 2.4.5: Suponhamos que $\phi$ seja um sistema monótono com tempo de vida $\tau \operatorname{IFR} \mid\left(\Im_{t}\right)$. Então as realizações do Processo de Risco $\left(A_{\phi}(t)\right)_{t \geq 0}$ são convexas q.c. em $t \in(0, \tau]$.

Prova: Seja $Q=\left\{\frac{k}{2^{n}}: k, n \in \mathbb{N}\right\}$. Como $A_{t}(\phi)$ é contínuo à direita e $Q$ é denso em $\mathbb{R}^{+}$, é suficiente provar que

$$
A_{t+h}(\omega ; \phi)-A_{t}(\omega ; \phi) \geq A_{t^{*}+h}(\omega ; \phi)-A_{t^{*}}(\omega ; \phi)
$$

para todo $t, t^{*}$ e $h \in Q$ tais que $0 \leq t^{*} \leq t \leq t+h \leq \tau(\omega)$, pois neste caso concluímos que $A_{t}(\omega, \phi)$ tem derivada crescente e, portanto, é convexa em $(0, \tau(\omega))$ (a convexidade em $\tau(\omega))$ segue desde que $A_{t}(\phi)$ é crescente e contínua à direita).

Para provar a desigualdade acima usaremos que o compensador $A_{t}(\phi)$ é calculável (Teorema A.2.1). Esta propriedade assegura que $B_{n}(t) \rightarrow A_{t}(\phi)$ em probabilidade quando $n \rightarrow \infty$ onde $B_{n}(t)=\sum_{\frac{j}{2^{n}}<t} \mathbb{P}\left\{\frac{j}{2^{n}}<\tau \leq \frac{j+1}{2^{n}} \mid \Im_{\frac{j}{2^{n}}}\right)$.

Se $\mathbb{P}^{*}\left(\frac{j}{2^{n}}<\tau \leq \frac{j+1}{2^{n}} \mid \Im_{\frac{j}{2^{n}}}\right)$ é uma versão regular de $\mathbb{P}\left(\frac{j}{2^{n}}<\tau \leq \frac{j+1}{2^{n}} \mid \Im_{\frac{j}{2^{n}}}\right)$ e $B_{n}^{*}(t)$ é a respectiva versão de $B_{n}(t)$, existe um conjunto de medida nula $N_{0}$ e uma subsequência $\left(B_{n_{k}}^{*}(t)\right)_{k \geq 1}$, tal que se $\omega \notin N_{0}, B_{n_{k}}^{*}(\omega ; t) \rightarrow A_{t}(\omega ; \phi)$ quando $k \rightarrow \infty$. Consequentemente, para $\omega \notin N_{0}$ e $t, h \in Q$ vale:

$$
\begin{aligned}
A_{t+h}(\omega ; \phi)-A_{t}(\omega ; \phi) & =\lim _{k \rightarrow \infty}\left(B_{n_{k}}^{*}(\omega, t+h)-B_{n_{k}}^{*}(\omega ; t)\right) \\
& =\lim _{k \rightarrow \infty} \sum_{0 \leq \frac{j}{2^{n_{k}}}<h} \mathbb{P}^{*}\left(t+\frac{j}{2^{n_{k}}}<\tau \leq t+\frac{j+1}{2^{n_{k}}} \mid \Im_{t+\frac{j}{2^{n_{k}}}}\right) .
\end{aligned}
$$

Contudo $\tau$ é $\operatorname{IFR} \mid\left(\Im_{t}\right)$ e assim, no conjunto $\left\{t+\frac{j}{2^{n_{k}}}<\tau\right\}$ temos

$$
\begin{aligned}
\mathbb{P}^{*}\left(t+\frac{j}{2^{n_{k}}}<\tau \leq t+\frac{j+1}{2^{n_{k}}} \mid \Im_{t+\frac{j}{2^{n_{k}}}}\right) & =1-\mathbb{P}^{*}\left(\left(\tau-t-\frac{j}{2^{n_{k}}}\right)^{+}>\frac{1}{2^{n_{k}}} \mid \Im_{t+\frac{j}{2^{n_{k}}}}\right) \\
& \geq 1-\mathbb{P}^{*}\left(\left(\tau-t^{*}-\frac{j}{2^{n_{k}}}\right)^{+}>\frac{1}{2^{n_{k}}} \mid \Im_{t^{*}+\frac{j}{2^{n_{k}}}}\right) \\
& =\mathbb{P}^{*}\left(t^{*}+\frac{j}{2^{n_{k}}}<\tau \leq t^{*}+\frac{j+1}{2^{n_{k}}} \mid \Im_{t^{*}}+\frac{j}{2^{n_{k}}}\right) q . c .
\end{aligned}
$$

Desta maneira, existe um conjunto de medida nula $N_{k}$ tal que se $\omega \notin N_{k}$, vale 


$$
\begin{aligned}
& \sum_{0 \leq \frac{j}{2^{n_{k}}}<h} \mathbb{P}^{*}\left(t+\frac{j}{2^{n_{k}}}<\tau \leq t+\frac{j+1}{2^{n_{k}}} \mid \Im_{t+\frac{j}{2^{n_{k}}}}\right) \geq \\
\geq & \sum_{0 \leq \frac{j}{2^{n_{k}}}<h} \mathbb{P}^{*}\left(t^{*}+\frac{j}{2^{n_{k}}}<\tau \leq t^{*}+\frac{j+1}{2^{n_{k}}} \mid \Im_{t^{*}+\frac{j}{2^{n_{k}}}}\right)
\end{aligned}
$$

para todo $t, t^{*}, h \in Q$ tais que $0 \leq t^{*} \leq t \leq t+h \leq \tau(\omega)$. Assim, se $\omega$ não pertence ao conjunto nulo $N=N_{0} \cup\left(\bigcup_{k} N_{k}\right)$, concluímos que

$$
A_{t+h}(\omega ; \phi)-A_{t}(\omega ; \phi) \geq A_{t^{*}+h}(\omega ; \phi)-A_{t^{*}}(\omega ; \phi),
$$

como queríamos demonstrar.

A recíproca do teorema acima não é verdadeira, como mostra o seguinte contraexemplo

Exemplo 2.4.6: Consideremos um sistema monótono com tempo de vida $\tau$ com componentes $C_{1}$ e $C_{2}$. O componente $C_{1}$ tem tempo de vida $S_{1}$ com distribuição Exponencial de parâmetro 1 e o componente $C_{2}$ tem tempo de vida $S_{2}=S_{1}+Y$, com

$$
\mathbb{P}\left(Y \leq y \mid T_{1}\right)=1-e^{-\alpha\left(T_{1}\right) y}
$$

onde a função $\alpha(t)$ é estritamente decrescente e positiva. O tempo de vida do sistema é dado por $\tau=S_{2}=S_{1}+Y$.

Como $S_{1}$ nunca é crítica temos que $\sigma_{\phi}(1)=\infty$ e desde que $\left(Y \mid S_{1}\right)$ é exponencial de parâmetro $\alpha\left(S_{1}\right)$, concluímos que $\sigma_{\phi}(2)=S_{1}$. Assim,

$$
\begin{aligned}
A_{t}(\phi) & =\left[A_{t \wedge \tau}(1)-A_{\sigma_{\phi}(1)}(1)\right]^{+}+\left[A_{t \wedge \tau}(2)-A_{\sigma_{\phi}(2)}(2)\right]^{+} \\
& =\left[A_{t \wedge \tau}(1)-A_{\infty}(1)\right]^{+}\left[A_{t \wedge \tau}(2)-A_{S_{1}}(2)\right]^{+} \\
& =\left[A_{t \wedge \tau}(2)-A_{S_{1}}(2)\right]^{+} \\
& = \begin{cases}0, & \text { se } t \leq S_{1} \\
\alpha\left(S_{1}\right)\left(t \wedge \tau-S_{1}\right), & \text { se } t>S_{1} .\end{cases}
\end{aligned}
$$

Dessa forma, $A_{t}(\phi)$ é convexa. Por outro lado, no conjunto $\left\{S_{1}>t\right\}$, temos que 


$$
\begin{aligned}
\mathbb{P}\left(\left(S_{2}-t\right)^{+}>u \mid \Im_{t}\right) & =\mathbb{P}\left(\left(S_{2}-t\right)^{+}>u \mid S_{1}>t\right) \\
& =\mathbb{P}\left(\left(S_{1}+Y-t\right)^{+}>u \mid S_{1}>t\right) \\
& =\mathbb{P}\left(Y+\left(S_{1}-t\right)^{+}>u \mid\left(S_{1}-t\right)^{+}>0\right) \\
& =\int_{0}^{\infty} \mathbb{P}\left(Y>u-s \mid S_{1}=t+s\right) e^{-s} d s \\
& =\int_{0}^{\infty} e^{-\alpha(t+s)(u-s)^{+}} e^{-s} d s .
\end{aligned}
$$

Como $\alpha(t)$ é estritamente decrescente em $\left\{S_{1}>t\right\}$, concluímos que $\mathbb{P}\left((\tau-t)^{+}>\mid \Im_{t}\right)$ é crescente, de maneira que $\tau$ não é $\operatorname{IFR} \mid\left(\Im_{t}\right)$.

A conjectura de que a superaditividade do Processo de risco implica na propriedade $\operatorname{NBU} \mid\left(\Im_{t}\right)$ é falsa. No exemplo anterior, observamos que $A_{t}(\phi)$ é superaditiva. Entretanto

$$
\mathbb{P}\{\tau>u\}=\int_{0}^{\infty} e^{-\alpha(s)(u-s)^{+}} e^{-s} d s \leq \mathbb{P}\left((\tau-t)^{+}>u \mid \Im_{t}\right),
$$

ou seja, $\tau$ não é NBU|( $\left.\Im_{t}\right)$.

No próximo exemplo veremos que a condição de que $\tau$ seja $\operatorname{NBU} \mid\left(\Im_{t}\right)$ não implica que $A_{t}(\phi)$ seja superaditiva.

Exemplo 2.4.7: Seja $S_{1}$ um tempo de vida com distribuição $\operatorname{IP}\left(S_{1}=1\right)=\epsilon$ e $\mathbb{P}\left(S_{1}=2\right)=1-\epsilon$. Seja $S_{2}$ determinada pela taxa de falha

$$
\alpha(t)= \begin{cases}0 & \text { se } t \in(0,1] \\ M & \text { se } S_{1}=1 \text { e } t \in(1,2] \\ 1 & \text { se } S_{1}=2 \text { e } t \in(1,2] \text { e } \\ 1 & \text { se } t \in(2, \infty)\end{cases}
$$

onde $M>1$.

Tomemos $\tau=S_{2}$, então

$$
\mathbb{P}(\tau>t)=\left\{\begin{array}{cl}
1 & t \in(0,1) \\
\epsilon e^{-M(t-1)}+(1-\epsilon) e^{-(t-1)} & t \in[1,2) \\
e^{-(t-2)}\left(\epsilon e^{-M}+(1-\epsilon) e^{-1}\right. & t \in[2, \infty)
\end{array}\right.
$$




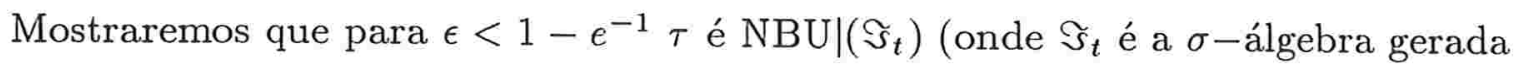
pelos indicadores dos componentes $)$, ou seja, $\mathbb{P}(\tau>t) \geq \mathbb{P}\left(\tau>t+s \mid \Im_{s}\right)$.

i) Se $s \in(0,1)$, para todo $t$

$$
\mathbb{P}\left(\tau>t+s \mid \Im_{s}\right)=\mathbb{P}(\tau>t+s) \leq \mathbb{P}(\tau>t)
$$

ii) Se $t \in(0,1)$, para todo $s$

$$
\mathbb{P}(\tau>t)=1 \geq \mathbb{P}\left(\tau>t+s \mid \Im_{s}\right)
$$

iii) Se $s \in[1,2)$ e $t \in[1, \infty)$,

$$
\begin{aligned}
& \mathbb{P}\left(\tau>t+s \mid S_{2}>s, S_{1}=1\right)=\frac{e^{-M} e^{-(t+s-2)}}{e^{-M(s-1)}} \leq e^{-t} \\
& \mathbb{P}\left(\tau>t+s \mid S_{2}>s, S_{1}=2\right)=e^{-t}
\end{aligned}
$$

iv) Se $s \in[2, \infty)$, para todo $t$

$$
\mathbb{P}\left(\tau>t+s \mid S_{2}>s, S_{1}\right)=e^{-t} .
$$

Assim, para $t \in[1, \infty)$ e $s \in[1, \infty)$

$$
\begin{aligned}
\mathbb{P}(\tau>t) & \geq e^{-(t-2)}\left(\epsilon e^{-M}+(1-\epsilon) e^{-1}\right) \\
& >e^{-(t-2)}(1-\epsilon) e^{-1} \\
& \geq e^{-t \quad} \quad\left(\text { se } \epsilon<1-e^{-1}\right) \\
& \geq \mathbb{P}\left(\tau>t+s \mid \Im_{s}\right) .
\end{aligned}
$$

Portanto, para $\epsilon<1-e^{-1} \tau$ é NBU|( $\left.\Im_{t}\right)$. Porém, em $\left\{S_{1}=1\right\}$ o $\left(\Im_{t}\right)$-compensador $A_{t}(\phi)$ tem a forma (q.c.)

$$
A_{t}(\phi)= \begin{cases}0 & \text { para } t \in(0,1), \\ M(t \wedge \tau-1) & \text { para } t \in[1,2) \mathrm{e} \\ M(2 \wedge \tau-1)+(t \wedge \tau-2)^{+} & \text {para } t \in[2, \infty) .\end{cases}
$$


Fixando $M=3$ no conjunto $\{\tau \geq 4\}$ temos

$$
A_{2}(\phi)=3 \text { e } A_{4}(\phi)=5
$$

portanto $A_{t}(\phi)$ não é superaditiva. 
APÊNDICE 


\section{A.1 Ordenação Estocástica.}

Denotaremos vetores do $\mathbb{R}^{n}$ por $\mathbf{x}=\left(x_{1}, x_{2}, \ldots, x_{n}\right)$. Dois vetores $\mathbf{x}$ e $\mathbf{y}$ são tais que $\mathbf{x}$ é menor do que $\mathbf{y}(\mathbf{x} \leq \mathbf{y})$ se e somente se $x_{i} \leq y_{i}, i=1, \ldots, n$.

Definição A.1.1: Um conjunto de Borel $U \subset \mathbb{R}^{n}$ é um conjunto superior se e somente se para quaisquer $\mathrm{x}$ e $\mathbf{y} \in \mathbb{R}^{n}, \mathrm{x} \leq \mathrm{y}$ e $\mathrm{x} \in U$ implicar $\mathrm{y} \in U$. Representaremos por $\mathcal{U}$ a classe de todos os conjuntos superiores.

Definição A.1.2: Se $\mathrm{X}=\left(X_{1}, X_{2}, \ldots, X_{n}\right)$ e $\mathrm{Y}=\left(Y_{1}, Y_{2}, \ldots, Y_{n}\right)$ são dois vetores aleatórios em $\mathbb{R}^{n}$, definidos em $(\Omega, \mathcal{A}, P)$ e $(\mathcal{W}, \Im, Q)$ respectivamente, dizemos que $\mathbf{X}$ é estocasticamente menor do que $\mathbf{Y}$ e denotaremos por $\mathbf{X} \leq(s t) \mathrm{Y}$, se e somente se

$$
\mathbb{P}(\mathrm{X} \in U) \leq Q(\mathrm{Y} \in U), U \in \mathcal{U}
$$

Uma caracterização útil para ordenação estocástica é dada pelo seguinte lema:

Lema A.1.3: $\mathbf{X} \leq(s t) \mathrm{Y}$ se e somente se $\mathbb{E}_{P}[f(\mathrm{X})] \leq \mathbb{E}_{Q}[f(\mathrm{Y})]$ para qualquer função $f: \mathbb{R}^{n} \rightarrow \mathbb{R}$, crescente, limitada e Borel mensurável.

\section{Definições A.1.4:}

- Ao conjunto superior $U_{\mathrm{x}}=\left(x_{1}, \infty\right) \mathrm{x}\left(x_{2}, \infty\right) \mathrm{x} \ldots \mathrm{x}\left(x_{n}, \infty\right), \mathrm{x} \in \mathbb{R}^{n}$, denominaremos corner superior.

- A classe $\mathcal{U}_{F}$ das uniões finitas de corner superiores denominaremos Domínio Superior Fundamental.

- Seja $\mathcal{U}_{Q}$ a subclasse de $\mathcal{U}_{F}$ formada pelas uniões finitas das $U_{\mathrm{x}} \operatorname{com} \mathrm{x} \in Q^{n}$, onde $Q$ é o conjunto dos números racionais.

- Seja $\mathcal{U}_{d}, d \in \mathbb{R}$, a subclasse de $\mathcal{U}_{F}$ formada pelas uniões finitas de conjuntos $U_{\mathrm{d}}$, onde $\mathrm{d}=\left(d_{1}, d_{2}, \ldots, d_{n}\right)$, com $d_{i}=d$ ou $d_{i}=-\infty$.

Claramente os conjuntos em $\mathcal{U}_{F}, \mathcal{U}_{Q}$ e $\mathcal{U}_{d}$ são abertos. 
Lema A.1.5: $U \in \mathcal{U}_{d}$ se e somente se existe um inteiro $1 \leq k_{0} \leq 2^{n}-1 \mathrm{e}$ subconjuntos $P_{1}, \ldots, P_{k_{0}}$ de $\{1,2, \ldots, n\}$ tais que $\mathbf{x} \in U$ se e somente se $\max _{1 \leq k \leq k_{0}} \min _{i \in P_{k}} x_{i}>d$.

\section{Prova:}

i) Se $\mathrm{x} \in U \in \mathcal{U}_{d}, \mathrm{x} \in U=\bigcup_{k=1}^{k_{0}} U_{\mathrm{d}^{k}}$ onde $\mathrm{d}^{k}=\left(d_{1}^{k}, d_{2}^{k}, \ldots, d_{n}^{k}\right)$ e $d_{i}^{k}=d$ ou $d_{i}^{k}=-\infty$. Sejam $P_{k}=\left\{i\right.$ tais que $\left.d_{i}^{k}=d\right\}, 1 \leq k \leq k_{0}$. Então $\mathrm{x} \in U_{\mathrm{d}^{k}}$ se e somente se $\min _{i \in P_{k}} x_{i}>d$ e $\mathbf{x} \in U=\bigcup_{k=1}^{k_{0}} U_{\mathrm{d}}^{k}$ se e somente se $\max _{1 \leq k \leq k_{0}} \min _{i \in P_{k}}>d$.

ii) inversamente, se $\mathbf{x}$ é tal que $\max _{1 \leq k \leq k_{0}} \min _{i \in P_{j}}>d$, então existe um $k, 1 \leq k \leq k_{0}$ tal que $\min _{i \in P_{k}} x_{i}>d$. Seja $\mathrm{d}^{k}$ tal que $d_{i}^{k}=d$ se $i \in P_{k}$ e $d_{i}^{k}=-\infty$ se $i \notin P_{k}$, de forma que

$$
\mathrm{x} \in \bigcup_{k=1}^{k_{0}} U_{\mathbf{d}^{k}} \in \mathcal{U}_{d}
$$

\section{Definições A.1.6:}

- A um conjunto $U \in \mathcal{U}_{d}, d \in \mathbb{R}$, denominaremos conjunto diagonal superior.

- A uma função $f: \mathbb{R}^{n} \rightarrow \mathbb{R}$ que depende de $\mathrm{x}$ somente através da relação $\max _{1 \leq k \leq k_{0}} \min _{i \in P_{k}} x_{i}$ onde $1 \leq k_{0} \leq 2^{n}-1$ é algum inteiro e $P_{1}, P_{2}, \ldots, P_{k_{0}}$ são subconjuntos de $\{\overline{1}, \overline{2}, \ldots, n\}$ denominaremos função diagonal.

Definição A.1.7: Dizemos que o vetor aleatório $\mathrm{X}=\left(X_{1}, \ldots, X_{n}\right)$ é estocasticamente menor do que o vetor aleatório $\mathrm{Y}=\left(Y_{1}, \ldots, Y_{n}\right)$, fracamente, se a classe do conjuntos superiores $\mathcal{U}$ na Definição A.1.2 for restrita à classe dos conjuntos superiores diagonais ou equivalentemente, se a classe das funçôes $f$ no Lema A.1.3 for restrita à das funções diagonais. 


\section{A.2 Compensadores Calculáveis.}

O $\left(\Im_{t}\right)$-compensador $A_{t}(I)$, onde $\Im_{t}=\sigma\left\{N_{s}(I), s \leq t, I \in \Lambda\right\}$, é calculável se é o limite em probabilidade de uma soma de probabilidades condicionais. Tecnicamente, $\left(Q_{n}\right)_{n \geq 1}$ é uma $R$-sequência de partições do intervalo $(0, s]$ se:

(1) Se $n \geq m$ então $Q_{n}$ é um refinamento de $Q_{m}$.

(2) $\max _{\left(t, t^{*}\right] \in Q_{n}}\left(t^{*}-t\right) \rightarrow 0$.

Seja $a\left(Q_{n}\right)=\sum_{\left(t, t^{*}\right] \in Q_{n}} \mathbb{E}\left[N_{t^{*}}(I)-N_{t}(I) \mid \Im_{t}\right]$. Dizemos que $A(I)$ é calculável se, para qualquer $s \geq 0$ e qualquer $R$-sequência de partições $\left(Q_{n}\right)_{n \geq 1}$ de $(0, s]$ tivermos

$$
a\left(Q_{n}\right) \rightarrow A_{s}(I)
$$

em probabilidade.

Dizemos que $A$ é calculável localmente (L-calculável) se o compensador $A_{t \wedge T_{k}}$ de $N_{t \wedge T_{k}}$ é calculável para cada $k$.

Teorema A.2.1 : Seja $\Im_{t}=\sigma\left\{N_{s}(I), s \leq t, I \in \Lambda\right\}$. O $\Im_{t}$-compensador $A_{t}(I)$ de $N_{t}(I)$ é L-calculável.

Para provar a proposição acima devemos usar os dois seguintes lemas

Lema A.2.2: $\operatorname{Se} \mathcal{G}$ e $\mathcal{H}$ são duas sub- $\sigma$-álgebras de $\mathcal{A}, B$ é um evento arbitrário, $C \in \mathcal{G}$ e $\mathcal{G} \cap C=\mathcal{H} \cap C$, então

$$
\mathbb{P}(B \cap C \mid \mathcal{G})= \begin{cases}\frac{\mathbb{P}(B \cap C \mid \mathcal{H})}{\mathbb{P}(C \mid \mathcal{H})}, & \text { se } \omega \in C^{C} \\ 0, & \text { se } \omega \in C^{C}\end{cases}
$$

onde $\mathbb{P}(C \mid \mathcal{H})$ é uma versão para a qual $\mathbb{P}(C \mid \mathcal{H})>0$ em $C$.

Prova: Seja $D \in \mathcal{G}$ 


$$
\begin{aligned}
\int_{D} \mathbb{P}\{B \cap C \mid \mathcal{G}) \mathbb{P}(d \omega) & \left.=\int_{D \cap C} \mathbb{P}(B \cap C \mid \mathcal{G}) \mathbb{P}(d \omega)+\int_{D \cap C^{c}} \mathbb{P}(B \cap C) \mid \mathcal{G}\right) \mathbb{P}(d \omega) \\
& =\int_{D \cap C} \frac{\mathbb{P}(B \cap C \mid \mathcal{H})}{\mathbb{P}(C \mid \mathcal{H})} P(d \omega) \\
& =\int_{D \cap C} \mathbb{P}(B \cap C \mid C \cap \mathcal{H}) \mathbb{P}(d \omega) \\
& =\int_{D \cap C} \mathbb{P}(B \cap C \mid C \cap \mathcal{G}) \mathbb{P}(d \omega) \\
& =\mathbb{P}(B \cap C \cap D) .
\end{aligned}
$$

Lema A.2.3: Para todo $k \geq 0$, e todo $t^{*}>t \geq 0$ temos

$$
\begin{aligned}
& \left.\mathbb{P}\left(\left\{T_{k}<t<T_{k+1} \leq t^{*}\right\} \cap\left\{X_{k+1}=I\right\}\right) \mid \Im_{t}\right)= \\
& = \begin{cases}\frac{\left.\mathbb{P}\left(\left\{t<T_{k+1} \leq t^{*}\right\} \cap\left\{I=X_{k+1}\right\} \mid \Im_{T_{k}}\right\}\right)}{\mathbb{P}\left(t<T_{k+1} \mid \Im_{T_{k}}\right)}, & \text { se } \omega \in\left\{T_{k}<t<T_{k+1}\right\} \\
0, & \text { c.c. }\end{cases}
\end{aligned}
$$

onde $\mathbb{I P}\left\{t<T_{k+1} \mid \Im_{T_{k}}\right)$ é uma versão não nula no conjunto $\left\{T_{k}<t<T_{k+1}\right\}$.

Prova: Considere $C=\left\{T_{k}<t<T_{k+1}\right\} \in \Im_{t}$. Note também que

$$
\left\{\left\{N_{t}(I)=r\right\} \cap\left\{T_{k}<t<T_{k+1}\right\}\right\}=\left\{\left\{N_{T_{k}}(I)=r\right\} \cap\left\{T_{k}<t<T_{k+1}\right\}\right\}
$$

de forma que $C \cap \Im_{t}=C \cap \Im_{T_{k}}$.

Podemos então considerar $\mathcal{G}=\Im_{t}, \mathcal{H}=\Im_{T_{k}}, B=\left\{T_{k+1} \leq t^{*}\right\} \cap\left\{X_{k+1}=I\right\}$ e $C=\left\{T_{k}<t<T_{k+1}\right\}$ e desde que $C \in G$, aplicar o Lema A.2.2.

Prova:(Teorema A.2.1). Sabemos que

$$
\begin{gathered}
A_{t}(I)=\sum_{k \geq 0} \int_{T_{k}}^{\imath \wedge T_{k+1}} \frac{G_{k}(d u, I)}{H_{k}[u, \infty)} . \\
-69-
\end{gathered}
$$


Consideremos para cada $k \geq 0$ o compensador.

$A_{s}^{k}(I)=A_{s \wedge T_{k+1}}(I)-A_{s \wedge T_{k}}(I)$ de $N_{s}^{k}(I)=N_{s \wedge T_{k+1}}(I)-N_{s \wedge T_{k}}(I)$

Seja $\left(Q_{n}\right)_{n \geq 1}$ uma $R$-sequência de partições de $(0, s]$. Se $t, t^{*} \in \mathbb{R}_{+}$, temos

$$
N_{t^{*}}^{k}(I)-N_{t}^{k}(I)=I_{\left\{t<T_{k+1} \leq t^{*}\right\}} I_{\left\{X_{k+1}=I\right\}}
$$

Assim,

$$
\begin{aligned}
a\left(Q_{n}\right)= & \sum_{\left(t, t^{*}\right\} \in Q_{n}} \mathbb{E}\left(N_{t^{*}}^{k}(I)-N_{t}^{k}(I) \mid \Im_{t}\right) \\
= & \sum_{\left(t, t^{*}\right] \in Q_{n}} \mathbb{E}\left(I_{\left\{t<T_{k+1} \leq t^{*}\right\}} I_{\left\{X_{(k+1)}=I\right\}} \mid \Im_{t}\right) \\
= & \sum_{\left(t, t^{*}\right] \in Q_{n}} \mathbb{E}\left(I_{\left\{t \leq T_{k}<T_{k+1} \leq t^{*}\right\}} I_{\left\{X_{k+1}=I\right\}} \mid \Im_{t}\right)+ \\
& \sum_{\left(t, t^{*}\right] \in Q_{n}} \mathbb{E}\left(I_{\left\{T_{k}<t<T_{k+1} \leq t^{*}\right\}} I_{\left\{X_{k+1}=I\right\}} \mid \Im_{t}\right) .
\end{aligned}
$$

Como

$$
\begin{aligned}
& \left\|\sum_{\left(t, t^{*}\right] \in Q_{n}} \mathbb{E}\left(I_{\left\{t \leq T_{k}<T_{k+1} \leq t^{*}\right\}} I_{\left\{X_{k+1}=I\right\}} \mid \Im_{t}\right)\right\|_{1}= \\
& =\sum_{\left(t, t^{*}\right] \in Q_{n}} \mathbb{P}\left(t \leq T_{k}<T_{k+1} \leq t^{*}, X_{k+1}=I\right) \rightarrow 0
\end{aligned}
$$

quando $n \rightarrow \infty$ temos

$$
a\left(Q_{n}\right) \sim \sum_{\left(t, t^{*}\right] \in Q_{n}} \mathbb{P}\left(T_{k}<t<T_{k+1} \leq t^{*}, X_{k+1}=I \mid \Im_{t}\right)
$$

. Utilizando o Lema A.2.3 concluímos que em $\left(T_{k}<t<T_{k+1}\right)$

$$
a\left(Q_{n}\right) \sim \sum_{\left(t, t^{*}\right] \in Q_{n}} \frac{\mathbb{P}\left(\left\{t<T_{k+1} \leq t^{*}\right\} \cap\left\{X_{k+1}=I\right\} \mid \Im_{T_{k}}\right)}{\mathbb{P}\left(t<T_{k+1} \mid \Im_{T_{k}}\right)}
$$

Para cada $\omega \in\left\{T_{k}<s\right\}$ podemos reconhecer que cada termo da soma do lado direito de A.2.1 é a integral da função $\sum_{\left(t, t^{*}\right) \in Q_{n}} I_{\left(t, t^{*}\right]}(u) \frac{1}{H_{k}\left[t^{*}, \infty\right)}$ com respeito à medida 
$G_{k}(d u, I)$, e concluímos, aplicando o teorema de convergência monótona para estas funções escadas, que para cada $\omega_{0} \in\left\{T_{k}<s\right\}$ obtemos

$$
a\left(Q_{n}\right) \rightarrow \int_{T_{k}}^{s \wedge T_{k+1}} \frac{G_{k}(d u, I)}{H_{k}[u, \infty)}=A_{s}(I)
$$

em probabilidade.

Corolário A.2.4: Em probabilidade, a seguinte convergência é válida

$$
\sum_{\frac{k}{2^{n}}<t} \mathbb{P}\left\{\frac{k}{2^{n}}<\tau_{\phi} \leq \frac{k+1}{2^{n}} \mid \Im_{\frac{k}{2^{n}}}\right\} \rightarrow A_{\phi}(t)
$$

Prova:

$$
\begin{aligned}
& \sum_{\frac{k}{2^{n}}<t} \mathbb{P}\left\{\frac{k}{2^{n}}<\tau_{\leq} \frac{k+1}{2^{n}} \mid \Im_{\frac{k}{2^{n}}}\right\}=\sum_{\frac{k}{2^{n}}<t} \sum_{I \in \Lambda} 1_{\left\{I \in \Gamma_{\phi}\left(\frac{k}{2^{n}}\right)\right\}} \mathbb{I P}\left\{\frac{k}{2^{n}}<\tau_{I} \leq \frac{k+1}{2^{n}} \mid \Im_{\frac{k}{2^{n}}}\right\} \\
& =\sum_{\frac{k}{2^{n}}<t} \sum_{I \in \Lambda} 1_{\left\{\sigma_{\phi}(I)<\frac{k}{2^{n}}<\tau \wedge t\right\}} \mathbb{P}\left\{\frac{k}{2^{n}}<\tau_{I} \leq \frac{k+1}{2^{n}} \mid \Im_{\frac{k}{2^{n}}}\right\} \\
& =\sum_{\frac{k}{2^{n}}<t} \sum_{\sigma_{\phi}(I)<\frac{k}{2^{n}}<\tau \wedge t} \mathbb{I P}\left\{\frac{k}{2^{n}}<\tau_{I} \leq \frac{k+1}{2^{n}} \mid \Im_{\frac{k}{2^{n}}}\right\} \\
& \rightarrow \sum_{I \in \Lambda}\left[A_{t \wedge \tau}(I)-A_{\sigma_{\phi}(I)}(I)\right]^{+} \\
& =A_{\phi}(t) \text {. }
\end{aligned}
$$




\section{BIBLIOGRAFIA}

[01] Arjas, E. (1981a) . A stochastic process approach to multivariate reability systems: notions based on conditional stochastic order. Mathematics of Operations Research, 6(2): 263-276.

[02] Arjas, E. (1981b). The failure and hazard process in multivariate reability systems. Mathematics of Operations Research, 6(4) : 551-562.

[03] Arjas, E. \& Norros, I. (1984) . Life lenghts and association: a dynamic approach. Mathematics of Operations Research, 9(1): 151-158.

[04] Barlow, R. \& Proschan, F. (1975). Importance of system components and fault tree events. Stochastic Process and their Application,3 :153-173.

[05] Barlow, R. \& Proschan, F. (1981). Statistical Theory of Reliability and Life Testing. To Begin Whith. Silver Spring, MD.

[06] Bergman, B. (1985) . On some new reliability importance measures IFAC. SAFECOMP W.J. Quirk Ed. Como, Italy, 61-64.

[07] Birnbaum, Z.W. (1969). On the importance of different components in a multicomponent system. Multivariate analysis. P.R. Krishnaiah Ed., Ny, 591-592.

[08] Brown, T. (1978). A martingale approach to the Poisson convergence of simple point processes. The Annals of Probability, 6(4): 615-628. 
[09] Jacod, J. (1975) . Multivariate point processes: predictable projection, RadonNikodym derivatives, representation of martingales. $Z$ Wahrscheinlichkeitstheorie Verw. Gebiete,31 : 235-253.

[10] Natvig, B. (1985). New light on measures of importance of systems components. Scandinavian Journal of Statistics, $12: 43-54$.

[11] Norros, I. (1986a) . Notes on Natvig's measure of importance of systems components. Journal of Applied Probability, 23 : 736-747.

[12] Norros, I. (1986b) . A compensator representation of multivariate life lenght distributions, with applications. Scandinavian Journal of statistics, 13 : 99-112.

[13] Liptser, R.S. \& Shiryayev A.N. (1977a). Statistics of Random Processes I . General Theory, Springer - Verlag, NY.

[14] Liptser, R.S. \& Shiryayev A.N. (1977b) . Statistics of Random Processes I . Applications, Springer - Verlag, NY. 